\title{
Thermal effects of nonuniform heating in a nanofluid-filled annulus: Buoyant transport versus entropy generation
}

\author{
M. Sankar ${ }^{1,2}$ (이 | H. A. Kumara Swamy ${ }^{2}$ (1) | Younghae Do Do $^{3}$ । \\ Sebastian Altmeyer ${ }^{4}$ (1)
}

${ }^{1}$ Department of General Requirements, University of Technology and Applied Sciences, Ibri, Oman

${ }^{2}$ Department of Mathematics, School of Engineering, Presidency University,

Bengaluru, Karnataka, India

${ }^{3}$ Department of Mathematics, KNU-Center for Nonlinear Dynamics, Kyungpook National University, Daegu, Republic of Korea

${ }^{4}$ Castelldefels School of Telecom and Aerospace Engineering, Universitat Politècnica de Catalunya, Barcelona, Spain

\section{Correspondence}

Younghae Do, Department of Mathematics, KNU-Center for Nonlinear Dynamics, Kyungpook National

University, Daegu 41566,

Republic of Korea.

Email: yhdo@knu.ac.kr

\section{Funding information}

National Research Foundation of Korea; VGST

\begin{abstract}
The primary challenge in the majority of heat transfer applications, in view of design perspective, is to maximize the thermal transport with the minimum generation of entropy. This paper addresses the numerical investigation of buoyant convection and entropy generation processes of $\mathrm{Al}_{2} \mathrm{O}_{3}$-water nanofluid inside a vertical annular configuration having two coaxial cylinders. The vertical cylindrical surfaces are imposed with sinusoidal thermal distribution having different phase deviation and amplitude, while the horizontal surfaces are retained adiabatic. In this analysis, the numerical computations of conservation equations governing the physical process are performed using the time-splitting technique and line overrelaxation methods. Detailed numerical simulations are performed for broad ranges of critical parameters, such as Rayleigh number, phase deviation, amplitude ratio, and nanoparticle volume fraction. From the vast numerical experiments, we systematically identified the suitable parameter regimes at which enhanced thermal transport has been produced with minimum entropy generation. Furthermore, the critical quantities, such as thermal dissipation and entropy generation, could be
\end{abstract}


effectively monitored by a particular selection of phase deviation $(\gamma)$ and amplitude ratio $(\varepsilon)$. It has been identified that these parameters have more influence on thermal transport as well as entropy production as compared to other parameters of the analysis.

\section{K E Y W O R D S}

annulus, entropy generation, nanofluid, natural convection, sinusoidal heating

\section{1 | INTRODUCTION}

Buoyant convective motion and associated thermal transport of traditional fluids and nanofluids in different finite-shaped enclosures are extensively analyzed through theoretical simulations and experimental visualizations. In particular, among the finite-sized geometries, the annular space formed by two up-right coaxial cylindrical tubes with different heating of side boundaries and insulated horizontal surfaces is considered as a suitable model problem aptly describing the physical configuration of many vital applications ranging from the cooling of electronic components to safety measures of nuclear reactors. The pioneering numerical attempt to explore the buoyant convection in an upright annular domain was by Davis and Thomas. ${ }^{1}$ They performed simulations for a broad spectrum of parameter ranges and suggested correlations for heat dissipation rate in various flow regimes. Also, Prasad and $\mathrm{Kulacki}^{2}$ initiated the experimental investigation of convective thermal transport of different convectional liquids in an annular enclosure with $\lambda=5.338$ and three aspect ratios. Considering the various need of applications, until recently, buoyant convective thermal transfer in an annular geometry was numerically investigated by considering various external constraints and different thermal boundary conditions. ${ }^{3-8}$ The inclusion of nanosized metals and oxides in traditional liquids was found to enhance the thermal transport rates and is corroborated through the predictions made by many theoretical simulations and experimental observations. ${ }^{9,10}$ Thermal transport analysis of different nanofluids in annular geometries has also received a great amount of attraction due to the requirement for effective cooling of electronic equipment. One of the earliest attempts to investigate the buoyant motion of $\mathrm{Al}_{2} \mathrm{O}_{3}$ nanofluid in an annular geometry is by Abouali and Falahatpisheh. ${ }^{11}$ Due to promising prospects of nanofluids in enhancing heat transport, numerous analysis of buoyant motion and thermal dissipation of nanofluids in an annulus has been investigated ${ }^{12-15}$ under different heating conditions and identified an appropriate thermal condition to enhance the heat dissipation rates. The boundary conditions have a major influence on buoyant flow rate and thermal transport rate was analyzed by several researchers. Natural convection with sinusoidal temperature profile with different phase deviations on the bounding surfaces was reported ${ }^{16-18}$ and concluded that nonuniform thermal conditions produce better heat dissipation rate than constant thermal conditions.

For the design of any heat transfer equipment, along with maximizing thermal dissipation rate, minimization of entropy generation is also an important parameter, since the efficiency of the system can be assessed by the produced entropy generation rate. Minimization of this 
undesirable quantity has been used as an important optimal design criterion for thermal systems. One of the pioneering analyses on buoyant transport and entropy generation in a rectangular geometry was by Ilis et al. ${ }^{19}$ by considering different aspect ratios. Chen et al. ${ }^{20}$ numerically investigated entropy production in an annular region and concluded that this quantity is an increasing function of Prandtl number. A numerical study was performed to study the thermal condition impacts on buoyant convection and entropy generation in a square geometry and reported the optimal condition to maximize thermal transport with minimal generation of entropy. ${ }^{21}$ Analysis of irreversibility generation in regular- and nonregularshaped porous enclosures has been numerically examined and reported the strategies to minimize the entropy production..$^{22-26}$ The impacts of discrete heating on convective motion and entropy generation in cavities were numerically investigated and reported that the location of heat sources plays a vital role in reducing the irreversibility production. ${ }^{27,28}$ Analysis of combined natural convection and entropy generation with surface radiation in a square cavity was studied and reported that enhancement of radiation emissivity reduces entropy generation. $^{29}$

Several investigations on buoyant transport and entropy production of infinite porous and nonporous geometries filled with a variety of nanofluids have been performed by considering effects, such as magnetic field and nonuniform thermal profiles. Shahi et al. ${ }^{30}$ numerically studied entropy production in a cavity filled with $\mathrm{Cu}-\mathrm{H}_{2} \mathrm{O}$ nanofluid containing protruded heater and found an optimum source location for maximum heat dissipation with minimum irreversibility. Alipanah et al. ${ }^{31}$ reported that the addition of $\mathrm{Al}_{2} \mathrm{O}_{3}$ nanoparticles to base fluid and increasing the Rayleigh number leads to enhancement of entropy production. Sheikholeslami et al. ${ }^{32}$ analyzed entropy production of different nanofluids in geometry containing heated objects and concluded that Ag-water nanofluid enhances both thermal dissipation rate and entropy generation simultaneously. Malik et al. ${ }^{33}$ applied the finite volume method and investigated that entropy generation of nanofluid increased with the enhancement of $R a$ and utilization of nanoparticles had a positive effect on heat transfer. The impact of nonuniform magnetic field on thermal dissipation and entropy production of nanofluid was numerically investigated by Mohammadpourfard et al. ${ }^{34}$ using a two-phase mixture model and control volume technique. Bezi et al. ${ }^{35}$ reported entropy production from the transient buoyant motion of nanofluid in a tilted semi-annular geometry and revealed that the irreversibility factor is sensitive to all critical parameter variations. Later, Kashyap and Dass ${ }^{36}$ numerically investigated entropy production generated by thermal transport of nanofluid-filled porous domain subjected to different nonuniform boundary conditions. Recently, Ferhi et al. ${ }^{37}$ analyzed the influence of magnetic field inclination angle and wall thickness on thermal transport and entropy generation of nanofluid in a discretely heated partitioned geometry.

In few applications, such as crystal growth processes and microgravity environments, the buoyant motion of nanofluids becomes undesirable and requires mechanisms to dampen such flow to design defect-free products. It has been proven that this unwanted fluid motion can be suppressed by employing the magnetic force of different strengths. Mejri et al. ${ }^{38}$ analyzed the effect of sinusoidal thermal profile in a nanofluid-filled square cavity and reported that for a particular choice of parameters heat transfer rate could be increased and entropy generation was decreased. Malik and Nayak ${ }^{39}$ analyzed the combined effects of discrete-time periodic sinusoidal thermal profile on buoyant motion of $\mathrm{Cu}$-water nanofluid in a porous geometry and identified a set of parameters at which the system performance could be enhanced. Recently, enhancement of thermal dissipation and minimization of entropy production in porous domain-containing hybrid nanofluids by considering magnetic field was also analyzed and the 
impacts are recorded. ${ }^{40}$ Buoyant nanofluid motion and associated thermal dissipation rates are highly sensitive to the shape of chosen geometry. In many practical applications, the geometrical configuration is not necessarily regularly shaped and the convective transport rates can be effectively controlled through vital geometrical parameters arising in nonregular geometries. By adopting the incompressible smoothed particle hydrodynamics method, Aly ${ }^{41}$ examined the control of buoyant motion of nanofluid in an H-shaped enclosure. In addition to heat transfer rate, irreversibility processes in a variety of odd-shaped geometries have also been significantly investigated by considering additional constraints. ${ }^{42-47}$ To consolidate the important predictions from various investigations on buoyant motion of nanofluids and entropy generation, a detailed review and discussions on different constraints influencing the buoyant transport and entropy production in finite geometries and passages have been discussed in detail. ${ }^{48-50}$

In the above discussions, a meticulous and systematic literature survey has been performed on the buoyant convection and irreversibility process of clear fluid and nanofluids in various shapes of finite geometries by considering the majority of additional constraints. From the detailed survey of theoretical and experimental investigations, we have noticed that the two important aspects, namely buoyant thermal transport and entropy behavior of nanofluids inside the enclosed annular geometry subjected to sinusoidal thermal profiles have not been analyzed so far. The knowledge of these two essential quantitative measures provides vital information on the performance of thermal systems. Many experimental and theoretical investigations on buoyant convective heat transfer and entropy production have been performed in finite-sized geometries for several decades. However, similar analyses have not been carried out in a nonuniformly heated annular geometry containing nanofluid. In view of the design perspective, the primary challenge in such applications is to maximize the thermal transport with the minimum generation of entropy to obtain better system efficiency. The existing results pertaining to the nonannular geometries cannot be utilized in many engineering applications represented by an annular geometry, such as cooling of electronic equipment, heat exchangers, nuclear reactors, and solar collectors. The apparent absence of this geometry, in spite of important applications, motivates the authors to perform a numerical investigation on buoyant convection and entropy generation in nanofluid-filled annulus subjected to nonuniform heating of the side walls. Therefore, a detailed numerical investigation is being carried out to identify the optimal key parameters to maximize thermal dissipation with subsidized the entropy production of $\mathrm{Al}_{2} \mathrm{O}_{3}-\mathrm{H}_{2} \mathrm{O}$ nanofluid in the annular geometry, which is most desirable in the design of any thermal system.

\section{2 | FORMULATION OF PROBLEM}

The geometrical design, as portrayed in Figure 1, is the enclosed concentric annulus region between two upright cylindrical tubes with $r_{i}$ and $r_{0}$ as radii of inner and outer cylindrical tubes. The vertical surfaces of cylindrical tubes are maintained with sinusoidal thermal distributions having different phase deviation and amplitude, while at the horizontal surfaces, the adiabatic condition is imposed. The annular gap is occupied by the water-based nanofluids having aluminum oxide $\left(\mathrm{Al}_{2} \mathrm{O}_{3}\right)$ nanoparticles. It is assumed that the water and nanoparticles are in thermal equilibrium. The gravity is chosen parallel to the $z$-axis, but in opposite direction. Also, the flow is considered to be unsteady, laminar, two-dimensional, and axisymmetric. Furthermore, the Boussinesq approximation, which accounts for the density variation in the body force 


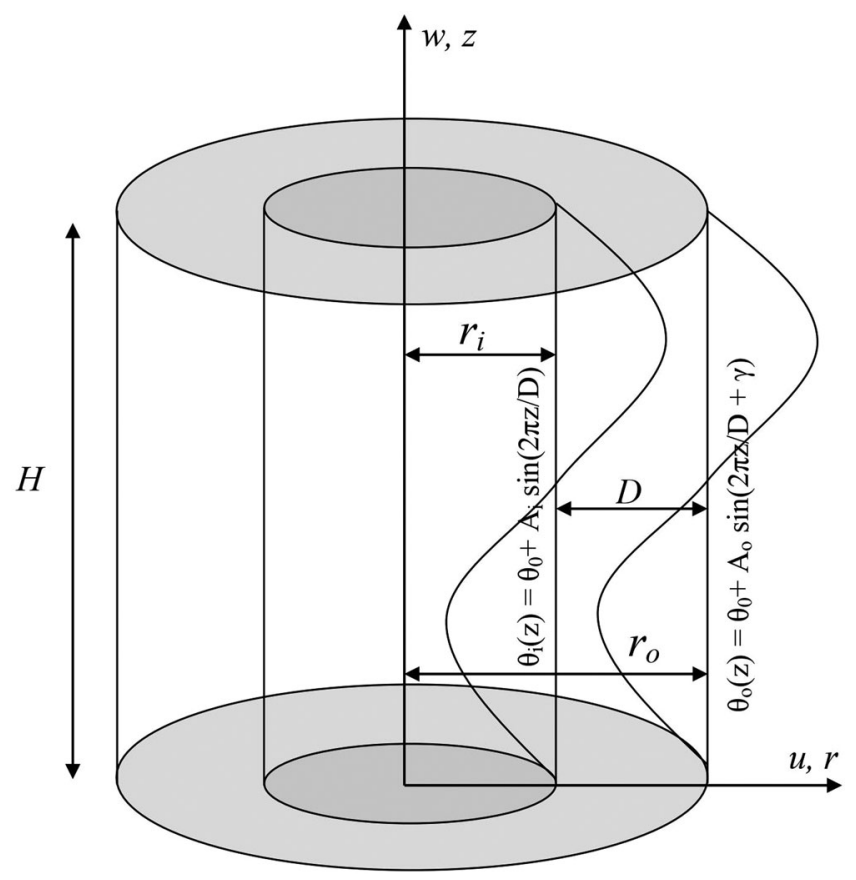

F I G URE 1 Geometrical configuration with thermal boundary conditions

term and constant elsewhere of momentum equation is utilized in this analysis. By utilizing the above postulates, the governing equations are: ${ }^{11,13}$

$$
\begin{gathered}
\nabla \cdot \vec{q}=0 \\
\rho_{n f}\left[\frac{\partial \vec{q}}{\partial t^{*}}+(\vec{q} \cdot \nabla) \vec{q}\right]=-\nabla p+\mu_{n f} \nabla^{2} q+(\rho \beta)_{n f} g\left(\theta-\theta_{0}\right), \\
\frac{\partial \theta}{\partial t^{*}}+(\vec{q} \cdot \nabla) \theta=\alpha_{n f} \nabla^{2} \theta .
\end{gathered}
$$

The following dimensionless variables are adopted in this analysis:

$$
(R, Z)=\frac{(r, z)}{D},(U, W)=\frac{(u, w)}{\alpha_{f} / D}, t=\frac{t^{*} \alpha_{f}}{D^{2}}, T=\frac{\left(\theta-\theta_{0}\right)}{\Delta \theta}, P=\frac{p D^{2}}{\rho_{n f} \alpha_{f}^{2}}
$$

By making use of the above transformations and eliminating pressure gradients from momentum equations, the nondimensional model equations in vorticity-stream function form are:

$$
\frac{\partial T}{\partial t}+U \frac{\partial T}{\partial R}+W \frac{\partial T}{\partial Z}=\frac{\alpha_{n f}}{\alpha_{f}} \nabla^{2} T
$$




$$
\begin{gathered}
\frac{\partial \zeta}{\partial t}+U \frac{\partial \zeta}{\partial R}+W \frac{\partial \zeta}{\partial Z}-\frac{U \zeta}{R}=\frac{\mu_{n f}}{\rho_{n f} \alpha_{f}}\left[\nabla^{2} \zeta-\frac{\zeta}{R^{2}}\right]-\frac{(\rho \beta)_{n f}}{\rho_{n f} \beta_{f}} \operatorname{Ra} \operatorname{Pr} \frac{\partial T}{\partial R}, \\
\zeta=\frac{1}{R}\left[\frac{\partial^{2} \psi}{\partial R^{2}}-\frac{1}{R} \frac{\partial \psi}{\partial R}+\frac{\partial^{2} \psi}{\partial Z^{2}}\right], \\
R U=\frac{\partial \psi}{\partial Z}, R W=-\frac{\partial \psi}{\partial R} .
\end{gathered}
$$

Here, $\nabla^{2}=\frac{\partial^{2}}{\partial R^{2}}+\frac{1}{R} \frac{\partial}{\partial R}+\frac{\partial^{2}}{\partial Z^{2}}, R a=\frac{g \beta_{f} \Delta \theta D^{3}}{v_{f} \alpha_{f}}$, and $P r=\frac{v_{f}}{\alpha_{f}}$ are Rayleigh and Prandtl numbers.

The auxiliary thermal conditions to solve Equations (4)-(6) are given below:

- At the vertical surface of inner cylinder:

$$
t>0: \psi=\frac{\partial \psi}{\partial R}=0, T=\sin (2 \pi Z) ; R=\frac{1}{\lambda-1} \text { and } 0 \leq Z \leq A r
$$

- At vertical surface of outer cylinder:

$$
t>0: \psi=\frac{\partial \psi}{\partial R}=0, T=\varepsilon \sin (2 \pi Z+\gamma) ; R=\frac{\lambda}{\lambda-1} \text { and } 0 \leq Z \leq A r
$$

- At horizontal surfaces of inner and outer cylinders:

$$
t>0: \psi=\frac{\partial \psi}{\partial Z}=0, \frac{\partial T}{\partial Z}=0 ; Z=0 \text { and } A r
$$

$A r=H / D$ is the aspect ratio, $\varepsilon=\frac{A_{o}}{A_{i}}$ is the amplitude ratio, $A_{i}$ and $A_{o}$ are, respectively, the amplitudes of interior and exterior cylinders, $\gamma$ is the phase deviation, and $\lambda=\frac{r_{o}}{r_{i}}$ is the radius ratio. The global heat transport across the annulus region is defined as the sum of average Nusselt numbers measured along the heating half of vertical boundaries and is estimated by $^{17,38}$

$$
\overline{N u}=\frac{1}{A r}\left[\int_{\text {heating half }} N u_{i} d Z+\int_{\text {heating half }} N u_{o} d Z\right]
$$

where $N u_{i}$ and $N u_{o}$ are the local Nusselt numbers estimated alongside the interior and exterior tubes, respectively, and are given below:

$$
N u_{i}=-\frac{k_{n f}}{k_{f}}\left(\frac{\partial T}{\partial R}\right)_{R=\frac{1}{\lambda-1}} \text { and } N u_{o}=-\frac{k_{n f}}{k_{f}}\left(\frac{\partial T}{\partial R}\right)_{R=\frac{\lambda}{\lambda-1}}
$$

The properties of nanofluid are estimated using the following correlations: ${ }^{38,39,49}$ 
Density: $\rho_{n f}=(1-\phi) \rho_{f}+\phi \rho_{p}$

Thermal expansion coefficient: $(\rho \beta)_{n f}=(1-\phi)(\rho \beta)_{f}+\phi(\rho \beta)_{p}$

Heat capacitance: $\left(\rho C_{p}\right)_{n f}=(1-\phi)\left(\rho C_{p}\right)_{f}+\phi\left(\rho C_{p}\right)_{p}$

Thermal conductivity: $\frac{k_{n f}}{k_{f}}=\frac{k_{p}+2 k_{f}-2 \phi\left(k_{f}-k_{p}\right)}{k_{p}+2 k_{f}+\phi\left(k_{f}-k_{p}\right)}$ (Maxwell Garnett's model)

Dynamic viscosity: $\mu_{n f}=\frac{\mu_{f}}{(1-\phi)^{2.5}}$ (Brinkman model)

Thermal diffusivity: $\alpha_{n f}=\frac{k_{n f}}{\left(\rho C_{p}\right)_{n f}}$

The thermophysical properties of water and nanoparticles are given in Table 1.

\section{3 | ENTROPY GENERATION EQUATION}

Based on the second law of thermodynamics and assumptions made, the dimensional entropy generation equation within the annulus is given by ${ }^{49}$

$$
S_{\text {gen }}=\frac{k_{n f}}{\theta_{0}^{2}}\left[\left(\frac{\partial \theta}{\partial r}\right)^{2}+\left(\frac{\partial \theta}{\partial z}\right)^{2}\right]+\frac{\mu_{n f}}{\theta_{0}}\left[2\left\{\left(\frac{\partial u}{\partial r}\right)^{2}+\left(\frac{u}{r}\right)^{2}+\left(\frac{\partial w}{\partial z}\right)^{2}\right\}+\left(\frac{\partial u}{\partial z}+\frac{\partial w}{\partial r}\right)^{2}\right]
$$

Utilizing the nondimensional transformations, the dimensionless local entropy generation can be expressed as

$$
S_{\mathrm{GEN}}=\frac{k_{n f}}{k_{f}}\left[\left(\frac{\partial T}{\partial R}\right)^{2}+\left(\frac{\partial T}{\partial Z}\right)^{2}\right]+\Phi \frac{\mu_{n f}}{\mu_{f}}\left[2\left\{\left(\frac{\partial U}{\partial R}\right)^{2}+\left(\frac{U}{R}\right)^{2}+\left(\frac{\partial W}{\partial Z}\right)^{2}\right\}+\left(\frac{\partial U}{\partial Z}+\frac{\partial W}{\partial R}\right)^{2}\right]
$$

where $\Phi=\frac{\mu_{f}}{k_{f}} \theta_{0}\left(\frac{\alpha_{f}}{D \Delta \theta}\right)^{2}$ is the irreversibility distribution ratio, $S_{\mathrm{GEN}}=\frac{S_{\mathrm{gen}} \theta_{0}^{2} D^{2}}{k_{f}(\Delta \theta)^{2}}$. The terms in Equation (9) can be rewritten in the following form:

$$
S_{\mathrm{GEN}}=S_{l . T}+S_{l . \Psi}
$$

Here, $S_{l . T}$ and $S_{l . \Psi}$ are local entropy generations due to thermal transport and fluid drag, respectively. The total/global entropy generation is calculated by integrating Equation (10) over the entire annular region and is given below:

TABLE 1 Thermophysical properties of water and nanoparticle ${ }^{32,33}$

\begin{tabular}{lll} 
Property & $\mathbf{H}_{\mathbf{2}} \mathbf{O}$ & $\mathbf{A l}_{\mathbf{2}} \mathbf{O}_{\mathbf{3}}$ \\
$\rho\left(\mathrm{kg} / \mathrm{m}^{3}\right)$ & 997.1 & 3970 \\
$C_{p}(\mathrm{~J} / \mathrm{kgK})$ & 4179 & 765 \\
$k(\mathrm{~W} / \mathrm{mK})$ & 0.613 & 40 \\
$\beta \times 10^{-5}\left(\mathrm{~K}^{-1}\right)$ & 21 & 0.85 \\
\hline
\end{tabular}




$$
\frac{1}{V} \int_{V} S_{\mathrm{GEN}} d V=\frac{1}{V} \int_{V} S_{l . T} d V+\frac{1}{V} \int_{V} S_{l . \Psi} d V
$$

The above equation can be represented as $S_{t o t}=S_{T}+S_{\Psi}$. An alternative parameter for irreversibility distribution is the Bejan number and is given by

$$
B e_{l}=\frac{S_{l . T}}{S_{l . T}+S_{l . \Psi}}
$$

The average Bejan number is obtained by integrating Equation (12) across the annular geometry and is estimated from the following expression.

$$
B e=\frac{1}{V} \int_{V} B e_{l} d V
$$

It is worth mentioning that if $B e \gg 0.5$ then $S_{T}$ is dominant, if $B e \ll 0.5$ then $S_{\Psi}$ is dominant and if $B e=0.5$, then the contribution of $S_{T}$ and $S_{\Psi}$ are equal. In addition, to measure the dominance of thermal dissipation over entropy generation or vice versa, the following quantity, namely performance evaluation criteria (PEC) is calculated. ${ }^{39,51}$ It is defined as $\Gamma=\overline{N u} / S_{\text {tot }}$.

\section{4 | SOLUTION PROCEDURE AND VALIDATION}

The coupled and nonlinear partial differential equations (PDEs) and associated auxiliary conditions governing the physical processes are numerically solved by utilizing a suitable implicit finite difference method (FDM) ${ }^{5,8,52}$ In particular, the transient PDEs, such as vorticity and temperature equations are discretized using alternating direction implicit method, and successive line overrelaxation technique is adopted to solve steady-state stream function equation. These FDM-based techniques reduce the PDEs to a system of linear algebraic finite difference equations of tridiagonal structure and using tridiagonal matrix algorithm, the solutions of these equations are obtained. Finally, Simpson and trapezoidal rules are, respectively, adopted to estimate the average Nusselt number and total entropy generation.

\section{1 | Grid sensitivity analysis}

In the present analysis, all numerical solutions are inspected for the grid independence trials by considering five different grid sizes, varying from sparse to finer grids, as shown in Table 2. The global Nusselt number and total entropy generation are monitored for each grid size and used as the sensitivity measure to check the optimum grid size. The difference on average Nusselt number and total entropy generation for a grid size of $201 \times 201$ and $251 \times 251$ is $\ll 0.1 \%$. Therefore, by accounting accuracy and computation time, a grid size of $201 \times 201$ is utilized for all simulations of the present investigation. An in-house FORTRAN code was written and few verifications are executed to validate the simulation results. 
TABLE 2 Grid independency study for $R a=10^{5}, \varepsilon=0.5, \gamma=\pi / 2$, and $\phi=0.05$

\begin{tabular}{lllll}
\hline Grid size & $\overline{\mathbf{N u}}$ & Relative difference & $\boldsymbol{S}_{\text {tot }}$ & Relative difference \\
\hline $81 \times 81$ & 4.9244237 & - & 219.2057983 & - \\
\hline $101 \times 101$ & 4.9237477 & 0.000756 & 219.0911086 & 0.0005232 \\
\hline $161 \times 161$ & 4.9122469 & 0.002341 & 218.8892694 & 0.0004648 \\
\hline $201 \times 201$ & 4.9116492 & 0.000121 & 218.8017605 & 0.0003997 \\
\hline $251 \times 251$ & 4.9112749 & 0.0000762 & 218.8004892 & 0.0000058 \\
\hline
\end{tabular}
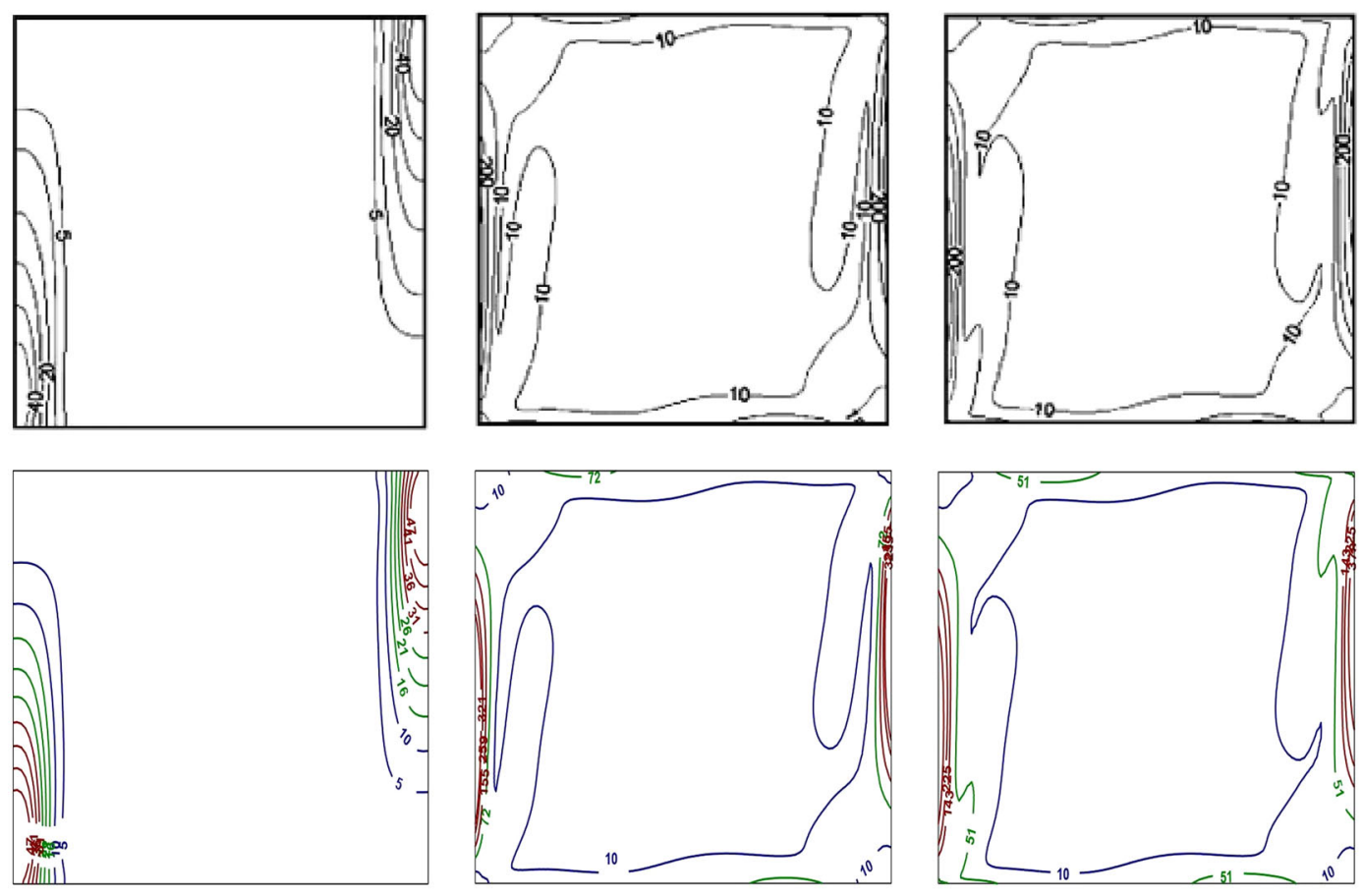

F I G U RE 2 Comparison of $S_{l . T}$ (left), $S_{l . \Psi}$ (middle), $S_{\mathrm{GEN}}$ (right) of present study (bottom) with Ilis et al. ${ }^{19}$ (top) for $R a=10^{5}, \Phi=10^{-4}$ [Color figure can be viewed at wileyonlinelibrary.com]

\section{2 | Validation}

To authenticate the present simulations, three qualitative and quantitative validations are performed with different benchmark data present in the literature. Initially, a qualitative comparison of flow and thermal contours in a square geometry containing conventional as well as nanofluids is generated by setting $\lambda=1$ in the present analysis. In first validation, our predictions on entropy generation for convectional fluid are compared with Ilis et al. ${ }^{19}$ The comparisons on entropy generation due to heat transfer, fluid friction, and total entropy generation, depicted in Figure 2, reveals fairly good agreement between both simulations. In the second comparison, for $\mathrm{Al}_{2} \mathrm{O}_{3}$-based nanofluid, the isotherms, streamlines, and total entropy generation are obtained from our code and are compared with those predictions of 
Mejri et al., ${ }^{38}$ and is shown in Figure 3. This comparison also reveals a very good qualitative agreement between the two investigations. Finally, the global Nusselt number for an annular geometry containing $\mathrm{Al}_{2} \mathrm{O}_{3}$-based nanofluid is compared with present predictions for the limiting case of uniform heating. The relative difference in $\overline{N u}$ between our predictions and those estimated from the correlation suggested by Abouali and Falahatpishesh, ${ }^{11}$ displayed in Table 3, is in the acceptable range for all magnitudes of $R a$. The excellent concurrence in the qualitative and quantitative comparison of our results with benchmark predictions for square and annular geometries ensures the credibility of the developed code.

\section{5 | RESULTS AND DISCUSSION}

In the present investigation, the buoyant thermal transport and entropy generation analysis is performed in the annular region whose walls are sinusoidally heated. The critical parameters of this analysis, varied over a wide spectrum of values, are Rayleigh number $\left(10^{3} \leq R a \leq 10^{5}\right)$, phase deviation $(0 \leq \gamma \leq \pi)$, amplitude ratio $(0 \leq \varepsilon \leq 1)$, and nanoparticle concentration $(0 \leq \phi \leq 0.05)$. For all simulations of the current study, the Prandtl number and irreversibility distribution ratio are fixed at $\operatorname{Pr}=6.2$ and $\Phi=10^{-3}$, respectively. Also, the geometrical variables such as radius ratio and aspect ratio are fixed to $\lambda=2$ and $A r=1$, respectively. The impact of these parameters on buoyant motion and production of entropy in the nanofluidfilled annular domain has been investigated qualitatively and quantitatively. The primary aim
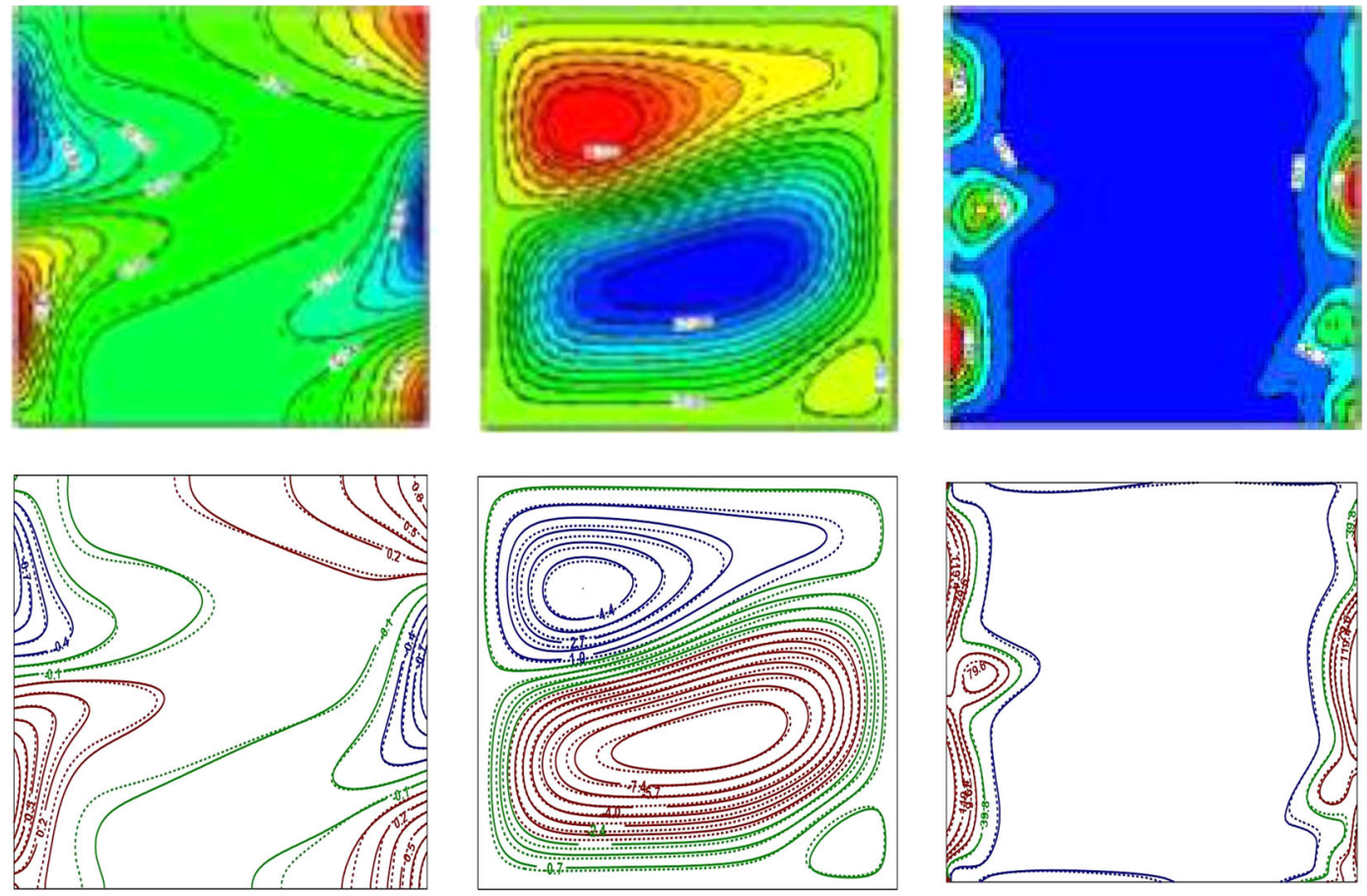

F I G U R E 3 Isotherms (left), streamlines (middle), $S_{\text {GEN }}$ (right) between present study (bottom) and Mejri et al. ${ }^{38}$ (top) for $R a=5 \times 10^{4}, \varepsilon=1, \gamma=\pi / 2 . \phi=0$ (dotted line), $\phi=0.04$ (solid line) [Color figure can be viewed at wileyonlinelibrary.com] 
TABLE 3 Comparison of $\overline{\mathrm{Nu}}$ of the present study with Abouali and Falahatpishesh ${ }^{11}$

\begin{tabular}{lllll}
$\boldsymbol{R a}(\mathbf{G r} \times \mathbf{P r})$ & $\begin{array}{l}\text { Abouali and } \\
\text { Falahatpishesh }^{11}\end{array}$ & Present study & $\begin{array}{l}\text { Relative } \\
\text { difference (\%) }\end{array}$ & \\
\hline $6 \times 10^{3}$ & 2.8673 & 2.8843 & 0.59 & $\phi=0.00$ \\
& 2.7291 & 2.7401 & 0.40 & $\phi=0.02$ \\
$6 \times 10^{4}$ & 5.4385 & 5.4796 & 0.75 & $\phi=0.00$ \\
& 5.1762 & 5.1836 & 0.14 & $\phi=0.02$ \\
\hline $6 \times 10^{5}$ & 10.3150 & 10.4015 & 0.83 & $\phi=0.00$ \\
& 9.8178 & 9.9132 & 0.96 & $\phi=0.02$ \\
\hline
\end{tabular}
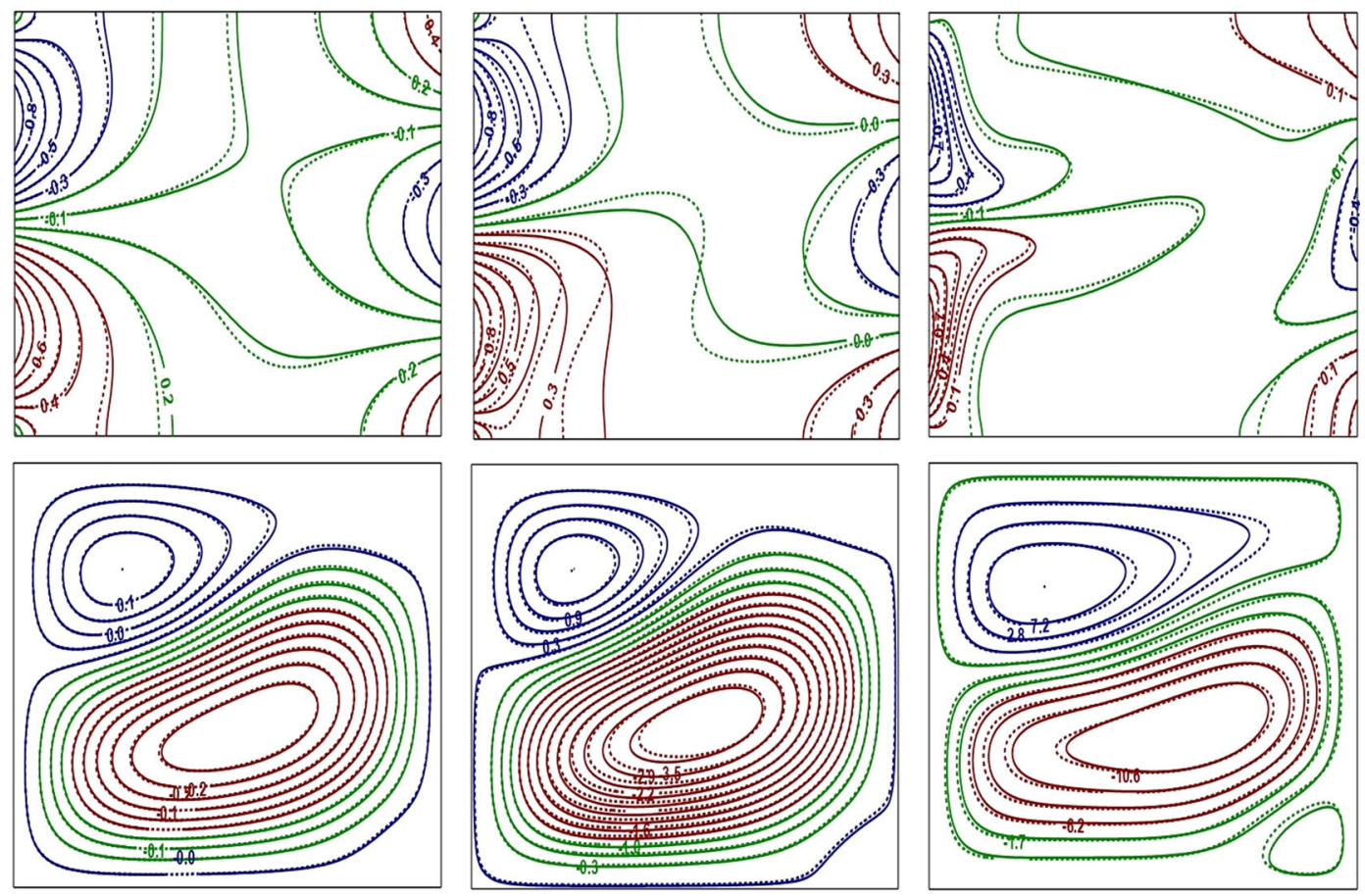

F I G URE 4 Isotherms (top) and streamlines (bottom) for different $R a$ at $\varepsilon=0.5, \gamma=\pi / 2, \phi=0$ (dotted line), $\phi=0.05$ (solid line). $R a=10^{3}$ (left), $R a=10^{4}$ (middle), and $R a=10^{5}$ (right) [Color figure can be viewed at wileyonlinelibrary.com]

of this numerical study is to illustrate the impacts of sinusoidal heating on thermal transport characteristics and entropy production in the annular region filled with $\mathrm{Al}_{2} \mathrm{O}_{3}-\mathrm{H}_{2} \mathrm{O}$ nanofluid. The flow and thermal contours are visualized through streamlines and isotherms. Assessment of thermal dissipation rate is evaluated in terms of $\overline{N u}$. Local and total entropy generation are displayed in the form of contours and bar graphs, respectively. Bejan number is determined to establish a clear picture of the overall contribution of individual entropy production to total entropy generation.

The effect of Rayleigh number on streamlines and isotherms for base fluid and nanofluid is depicted in Figure 4 by maintaining $\varepsilon$ and $\gamma$ at 0.5 and $\pi / 2$. At the lower Rayleigh number 
$\left(R a=10^{3}\right)$, streamlines exhibit two eddies with a smaller vortex on the top and a larger eddy at the bottom. The addition of nanoparticles has not altered the contour patterns appreciably and is similar for both base fluid and nanofluid. As $R a$ increases to $10^{4}$ no significant change in flow pattern is observed, due to the onset of convection from conductive transport. A further increase in Rayleigh number enhances the convective flow and isothermal stratification, and as a result, the size of the top eddy enhances and also leads to the generation of a new vortex at the right bottom. The isothermal pattern on both inner and outer cylinders are varying with an increase in Rayleigh number indicating that there is a significant change in heat transport with an enhancement of Rayleigh number. In general, the change in flow and thermal contours with $R a$ can be attributed to the nonuniform thermal conditions in the form of sinusoidal profiles, which is the main cause for multicellular flow generation.

Figure 5 deals with the isotherms and streamlines of the nanofluid and base fluid in the annular space for different phase deviations by keeping $\varepsilon$ and $R a$ as constant. In the absence of phase deviation, the streamlines exhibit four eddies with two small weak vortices near the outer cylinder and two large stronger eddies near the inner boundary. This variation in the size of eddies might be due to the larger amplitude at the inner boundary compared to the outer surface. In particular, the amplitude at the outer surface is only $25 \%$ of $\varepsilon$ at the inner boundary. As the phase deviation is increased, the secondary diagonal eddies are merged and there is a reduction in the size of primary diagonal eddies as well. A further increase in the phase deviation $(\gamma=\pi)$ leads to the elimination of the right bottom eddy and the formation of two larger eddies of the same size, which are symmetrical along $Z=A r / 2$. In general, with an increase in phase deviation, the number of eddies for both base fluid and nanofluid are reduced. As the magnitude of $\gamma$ increases, the isotherm pattern along the inner cylinder has not been changed appreciably; however, isotherms along the outer cylinder have undergone profound change as the variation in $\gamma$ affects the outer boundary.

The effect of $\varepsilon$ on the isotherms and streamlines of the base fluid and nanofluid can be seen in Figure 6 by fixing the phase deviation and Rayleigh number. For zero amplitude ratio, the streamlines exhibit two symmetrical eddies and isotherms show that maximum thermal energy is shifted from hotter to the colder region of the inner cylinder. The streamlines and isotherms are symmetric about their mid-height. This could be attributed to the fact that for $\varepsilon=0$, it is worth mentioning that the outer cylinder is maintained uniformly at a colder temperature. Enhancement of amplitude ratio destroys the symmetry with a decrease in the size of the upper vortex and an increase of bottom eddy size. A further rise in the magnitude of $\varepsilon$ leads to the formation of a new vortex at the right bottom of the annulus and increases its size. The variation in fluid flow and thermal pattern of nanofluid is also observed to be the same as of the base fluid; however, the magnitude of nanofluid motion decreases as compared to the base fluid. It has been noticed that the flow circulation strength estimated by $\left|\psi_{\max }\right|=7.67$ for base fluid $(\phi=0)$ reduces to $\left|\psi_{\max }\right|=7.06$ for nanofluid with $\phi=0.05$. Due to a particular choice of phase deviation $(\gamma=\pi / 2)$, it is noticed that higher heat transport takes place in the enclosure for unit amplitude ratio among others.

The entropy contours, such as $S_{l . T}, S_{l . \Psi}$, and $S_{\mathrm{GEN}}$ for different $R a$ has been discussed in Figure 7 for base fluid as well as nanofluid by keeping $\varepsilon=0.5$ and $\gamma=\pi / 2$. For the lower Rayleigh number, contours of $S_{l . T}$ are in wavy form near both the cylinders. On comparing the maximum entropy, measured by $S_{\max }$, for fluid and heat, it has been found that $S_{\max }(b f)=269.5$ due to heat transfer has been comparatively higher than $S_{\max }(b f)=1.3$ due to fluid friction. This indicates that the maximum entropy produced inside the annular region is mainly contributed from heat transfer and this phenomenon is true for nanofluids too. It can 

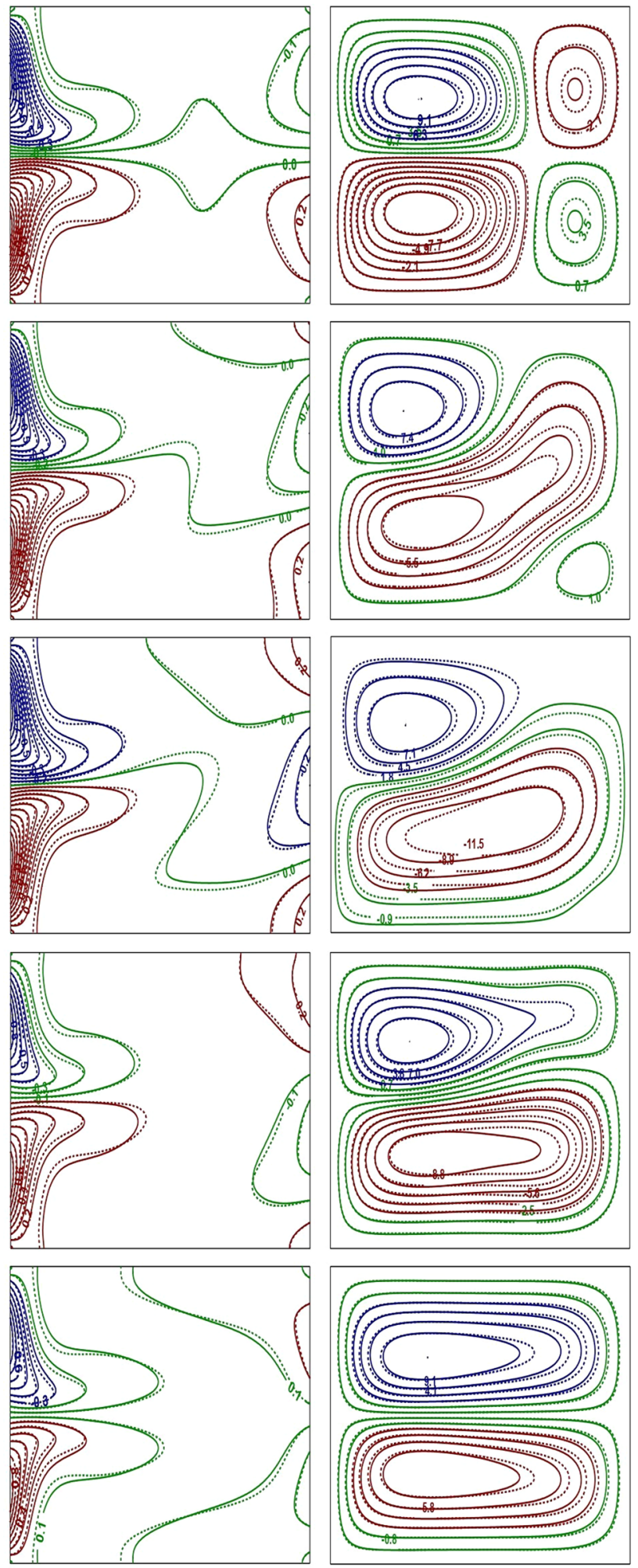

FIG URE 5 (See caption on next page) 
also be justified graphically that the contours of $S_{\mathrm{GEN}}$ look similar to that of $S_{l . T}$ or $S_{l . \Psi}$. It is interesting to note that the contour structure of $S_{\mathrm{GEN}}$ strongly depends on the patterns of $S_{l . T}$ and $S_{l . \Psi}$ along with the magnitude of $R a$. In particular, the structure of $S_{\mathrm{GEN}}$ is akin to that of $S_{l . T}$ for $R a=10^{3}$, while it is similar to $S_{l . \Psi}$ for $R a=10^{5}$ and is combined superimposed images of both $S_{l . T}$ and $S_{l . \Psi}$ for $R a=10^{4}$. This is consistent with the fact that heat transfer and fluid friction, respectively, contribute to entropy generation at lower and higher magnitudes of $R a$. Total entropy production in the enclosure has been reduced in the case of nanofluid compared to base fluid and can be seen through the relation $S_{\max }(n f)=257.47<S_{\max }(b f)=269.59$. Further increase in $R a$ to $10^{5}$, flow strength in the enclosure is enhanced, which leads to an increase in $S_{l . \Psi}$. In this case, fluid friction is a significant contributor to entropy generation in the annulus.

Local entropy generation due to heat transfer, fluid friction, and their combination for base fluid and nanofluid $(\phi=0.05)$ at different phase deviations are presented in Figure 8 by fixing $R a=10^{5}$ and $\varepsilon=0.25$. In general, due to the higher Rayleigh number, the convective mode is dominant in all the cases due to which fluid friction irreversibility contributes more to total entropy generation in an enclosure. As illustrated in the contours, entropy generation is more packed near the inner boundary than the outer surface of the enclosure. This can be due to differences in the amplitude of sinusoidal thermal profiles along the vertical surfaces. For zero phase deviation, $S_{\max }(b f)=8295.34$ and $S_{\max }(n f)=6384.20$ but with an increase in phase deviation $(\gamma=\pi), S_{\max }(b f)=9477.05$ and $S_{\max }(n f)=7275.25$. Thus, it can be concluded that the enhancement of phase deviation increases the total entropy production. The contour pattern of entropy generation near the inner cylinder appears to be similar for all phase deviations but a change in contour pattern can be observed near the outer cylinder due to varying phase deviations.

Figure 9 shows the distribution of local entropy generation due to heat, fluid friction, and both by varying the amplitude ratio. Entropy contours appear to be similar for base fluid and nanofluid at all amplitude ratios. No entropy generation is noticed at the outer cylinder for zero amplitude ratio. Enhancing the amplitude ratio leads to the existence and increase of entropy contours at the outer wall. However, no significant change in the contour pattern of the inner wall is observed. Entropy due to friction is higher than heat transfer for all amplitude ratios due to strong convective motion. It is noted that the entropy contour structure due to friction is similar to the entropy generation due to both fluid and heat, indicating that $S_{l . \Psi}$ has more influence in entropy production than $S_{l . T}$.

Figure 10 illustrates the impact of $\gamma$ and $\varepsilon$ on the overall thermal dissipation rate for a fixed amplitude ratio $(\varepsilon=0.25)$ for base fluid and nanofluid. In general, $\overline{N u}$ enhances with an increment of $R a$ at all values of $\gamma$. From the results (Figure 10A), it can be observed that the thermal dissipation rate could be considerably enhanced with nanofluids compared to traditional fluid due to the higher thermal conductivity of nanofluids. The influence of phase deviation $(\gamma)$ on the thermal dissipation rate greatly depends on $R a$. For $R a \leq 10^{4}$, maximum heat is dissipated at $\gamma=\pi / 2$. However, for $R a>10^{4}$, maximum heat transport attains when $\gamma=3 \pi / 4$ (Figure 10A). Beyond these values of $\gamma$, the average $N u$ decreases. This reduction in

F I G URE 5 Isotherms and streamlines for different $\gamma$ at $\varepsilon=0.25, R a=10^{5} . \phi=0$ (dotted line), $\phi=0$. 05 (solid line). $\gamma=0$ (row-I), $\gamma=\pi / 4$ (row-II), $\gamma=\pi / 2$ (row-III), $\gamma=3 \pi / 4$ (row-IV), and $\gamma=\pi$ (row-V)

[Color figure can be viewed at wileyonlinelibrary.com] 

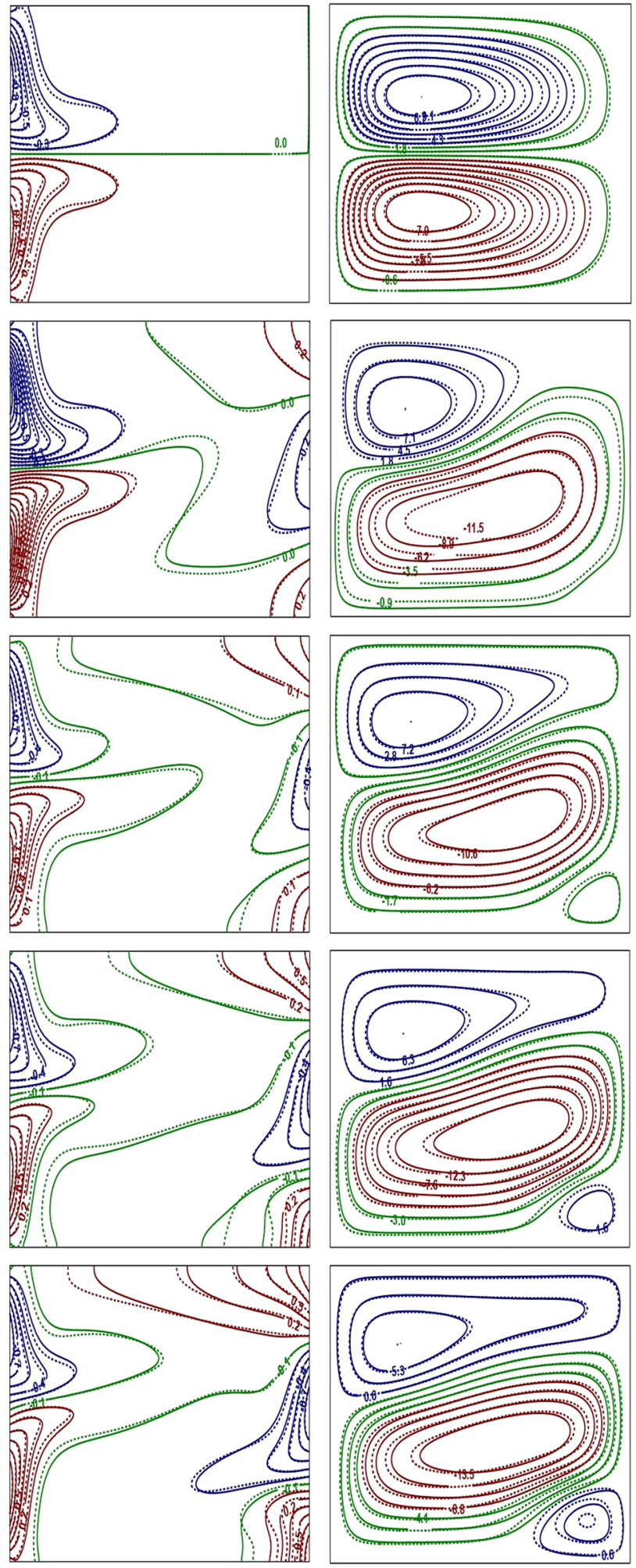

FIG URE 6 (See caption on next page) 

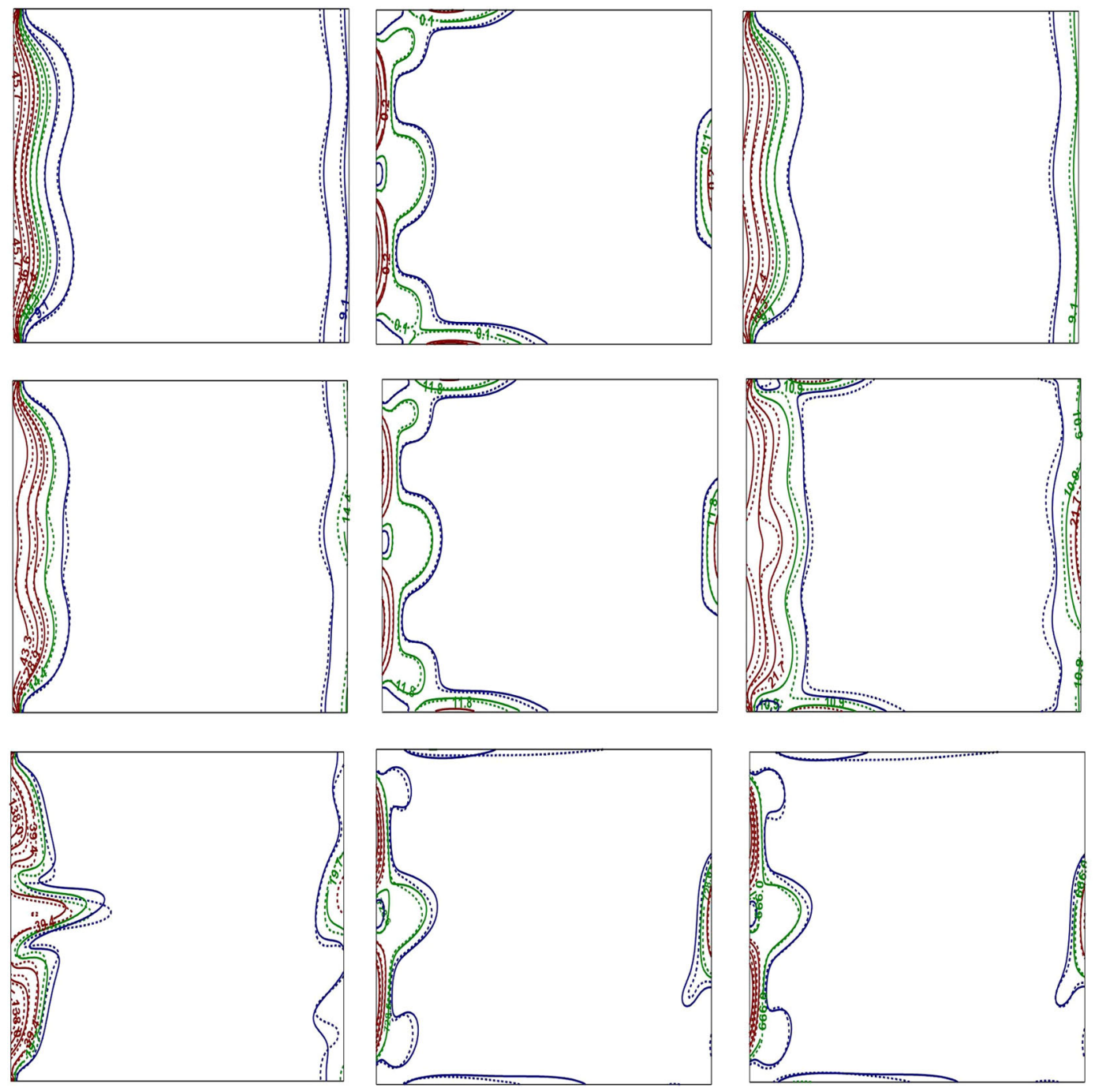

F I G U RE 7 Effect of $R a$ on $S_{l . T}$ (left), $S_{l . \Psi}$ (middle), $S_{\text {GEN }}$ (right) for $\varepsilon=0.5, \gamma=\pi / 2, \phi=0$ (dotted line), $\phi=0.05$ (solid line), $R a=10^{3}$ (row-I), $R a=10^{4}$ (row-II), and $R a=10^{5}$ (row-III) [Color figure can be viewed at wileyonlinelibrary.com]

thermal dissipation rate could be due to an increase in the phase deviation from 0 to $\pi$, which shifts the heating region on the right wall toward the top and the cooling region tends to shift toward the bottom portion. Due to this change, the thermal gradient and velocity will drops beyond $\gamma=3 \pi / 4$ and $\gamma=\pi / 2$, respectively, for $R a=10^{5}$ and $10^{3}$. A similar observation has also been predicted by Deng and Chang ${ }^{17}$ for natural convection in a rectangular enclosure

FIG URE 6 Isotherms and streamlines for different $\varepsilon$ at $\gamma=\pi / 2, R a=10^{5} . \phi=0$ (dotted line), $\phi=0.05$ (solid line) $\varepsilon=0$ (row-I), $\varepsilon=0.25$ (row-II), $\varepsilon=0.5$ (row-III), $\varepsilon=0.75$ (row-IV), and $\varepsilon=1$ (row-V) [Color figure can be viewed at wileyonlinelibrary.com] 

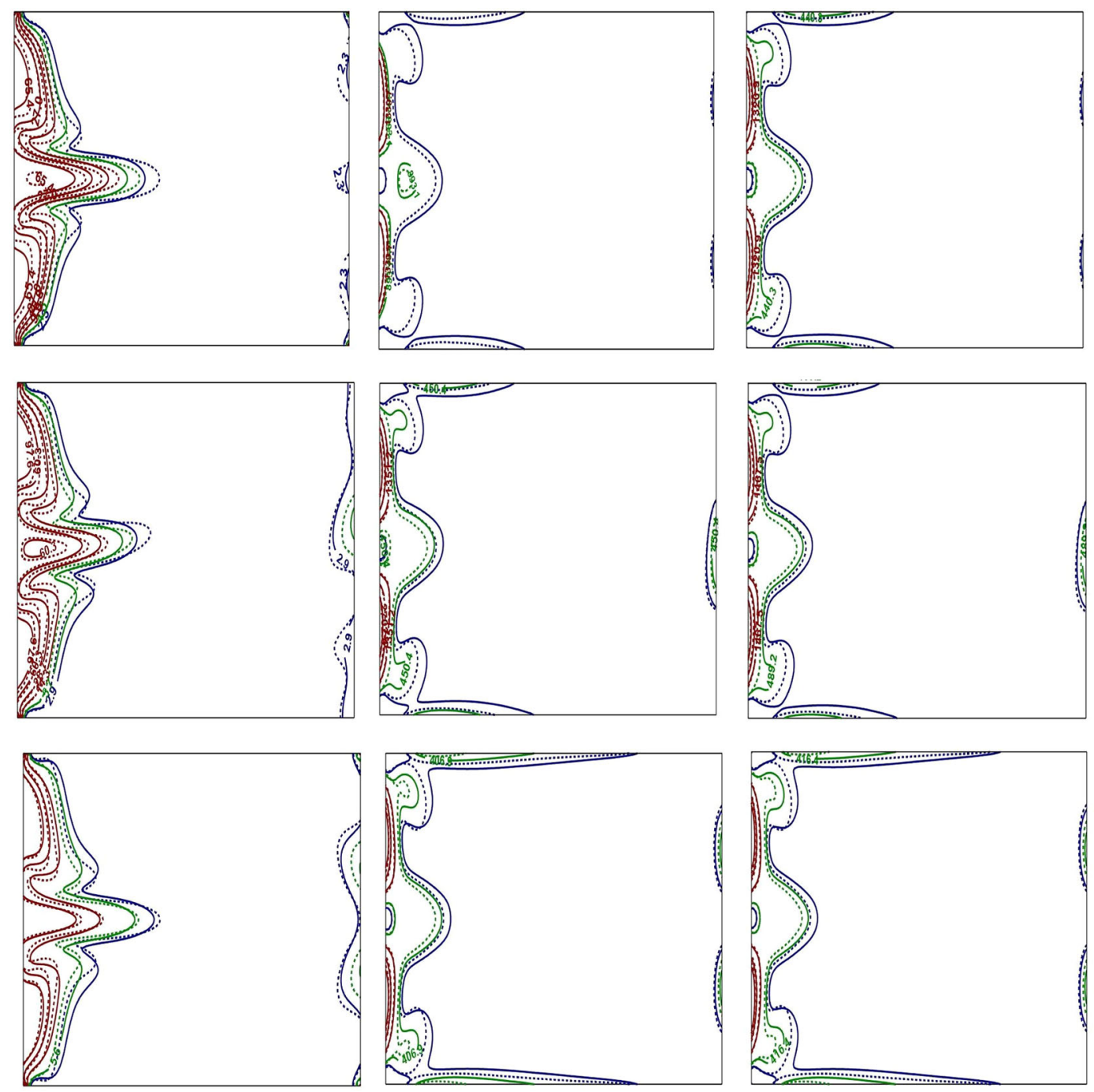

FIG URE 8 Effect of $\gamma$ on $S_{l . T}$ (left), $S_{l . \Psi}$ (middle), $S_{\mathrm{GEN}}$ (right) for $\varepsilon=0.25, R a=10^{5}, \phi=0$ (dotted line), $\phi=0.05$ (solid line). $\gamma=0$ (row-I), $\gamma=\pi / 2$ (row-II), $\gamma=\pi$ (row-III) [Color figure can be viewed at wileyonlinelibrary.com]

subjected to sinusoidal thermal distribution on both walls. Figure 10B deals with the effect of $\varepsilon$ and $\mathrm{Ra}$ on global $\mathrm{Nu}$ for both base fluid as well as nanofluid $(\phi=0.05)$ by maintaining phase deviation at $\pi / 4$. It is noted that the thermal transport rate in an enclosure, for this particular selection of parameters, is an increasing function of Rayleigh number and nanoparticle concentration. For all amplitude ratios, nanofluid has a better thermal dissipation rate as compared to the base fluid. Enhancement of amplitude ratio leads to a substantiate increment in $\overline{N u}$.

Figure 11 depicts the effect of nanoparticle concentration and Rayleigh number on global thermal transfer rate for fixed values of $\gamma$ and $\varepsilon$. As expected, enhancement of nanoparticle volume fraction leads to an increase in thermal conductivity of the fluid, which results in maximizing the heat transfer rate. Hence, on increasing the nanoparticle volume fraction, $\overline{\mathrm{Nu}}$ increases for all Rayleigh numbers. For $R a=10^{3}$ and $10^{4}$, it can be noticed that no significant 

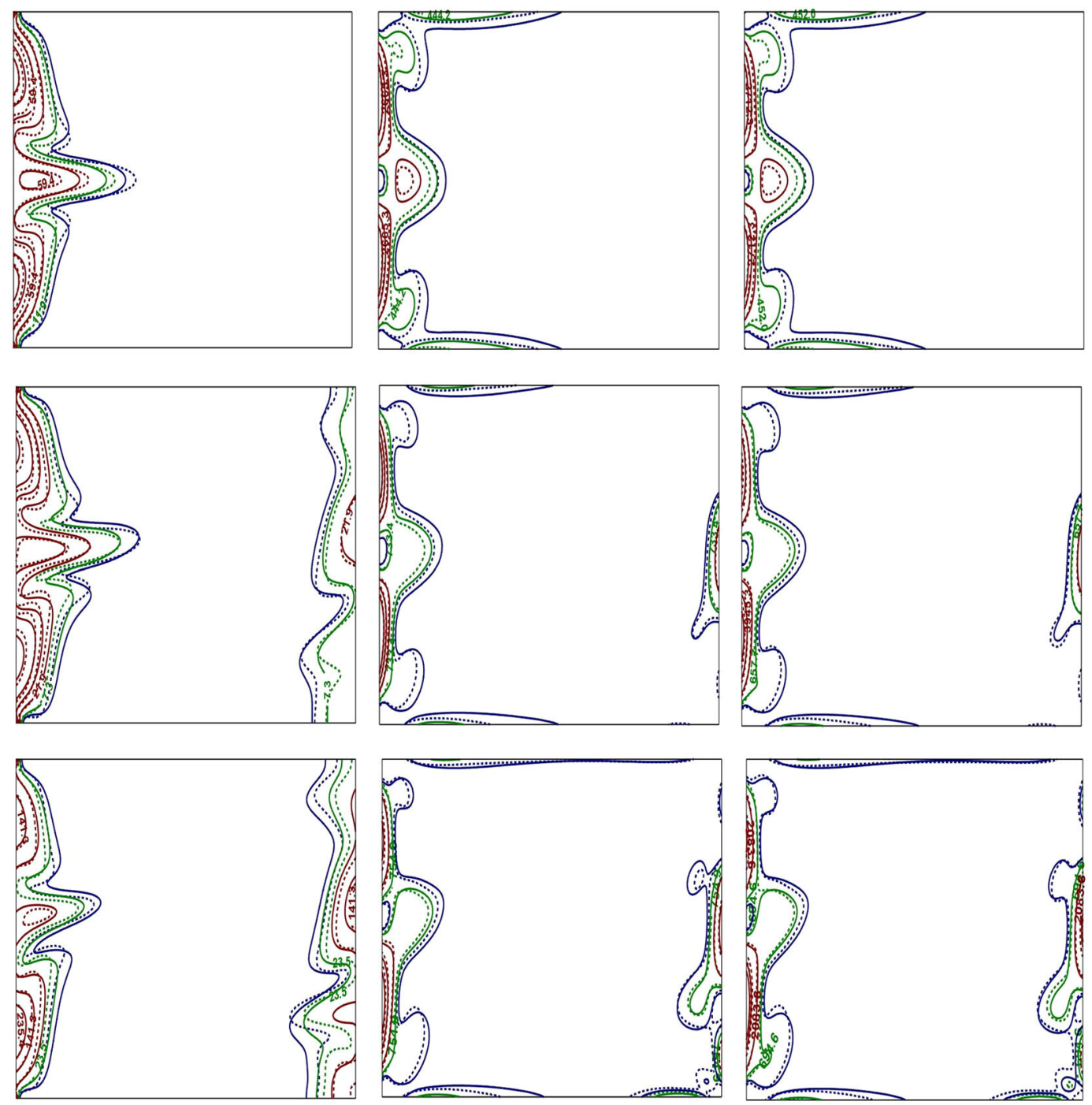

F I G U RE 9 Effect of $\varepsilon$ on $S_{l . T}$ (left), $S_{l . \Psi}$ (middle), $S_{G E N}$ (right) for $\gamma=\pi / 2, R a=10^{5} . \phi=0$ (dotted line), $\phi=0.05$ (solid line). $\varepsilon=0$ (row-I), $\varepsilon=0.5$ (row-II), $\varepsilon=1$ (row-III). [Color figure can be viewed at wileyonlinelibrary.com]

variation in thermal dissipation rate has been observed for higher nanoparticle concentrations. The impact of phase deviation and amplitude ratio on the average $\mathrm{Nu}$ of nanofluid $(\phi=0.05)$, at lower and higher values of $R a\left(R a=10^{3}\right.$ and $\left.R a=10^{5}\right)$, are predicted in Figure 12. For zero amplitude ratio, phase deviation has no influence on heat transport due to uniform cooling thermal conditions at the outer boundary. As a result, $\overline{\mathrm{Nu}}$ remains invariant with an increase in phase deviation at both the Rayleigh numbers. For each value of phase deviation, an increase in amplitude ratio results in the enhancement of $\overline{N u}$. For any value of $\varepsilon>0$, maximum thermal transfer rate takes place at $\gamma=\pi / 2$ for $R a=10^{3}$ and $\gamma=3 \pi / 4$ for $R a=10^{5}$. A similar observation has also been detected in Figure 10a. A closer observation on the impacts of $\gamma$ and $\varepsilon$ on global $N u$ reveals an important prediction that the thermal dissipation rate could be 

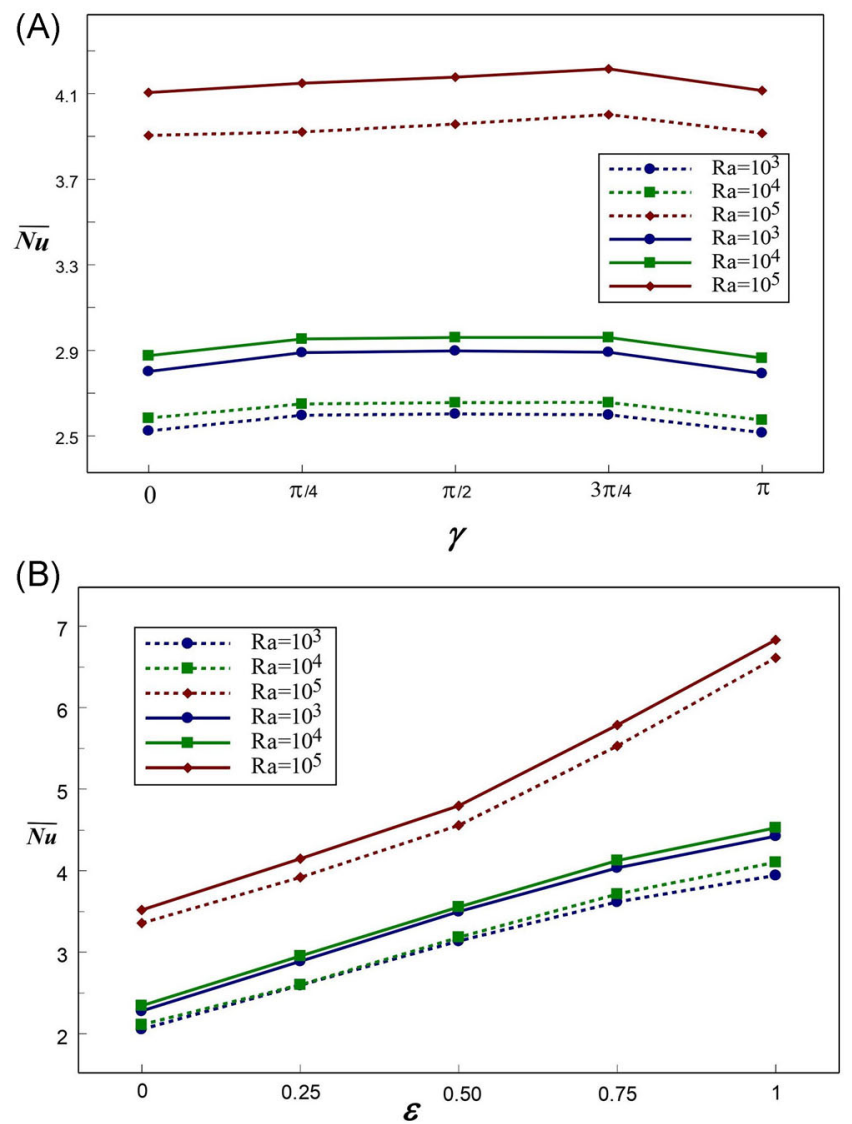

F I G U RE 10 Influence of various $R a$ on $\overline{N u}$ with (A) phase deviation $(\gamma)(\mathrm{B})$ amplitude ratio $(\varepsilon), \phi=0$ (dotted line), $\phi=0.05$ (solid line) [Color figure can be viewed at wileyonlinelibrary.com]

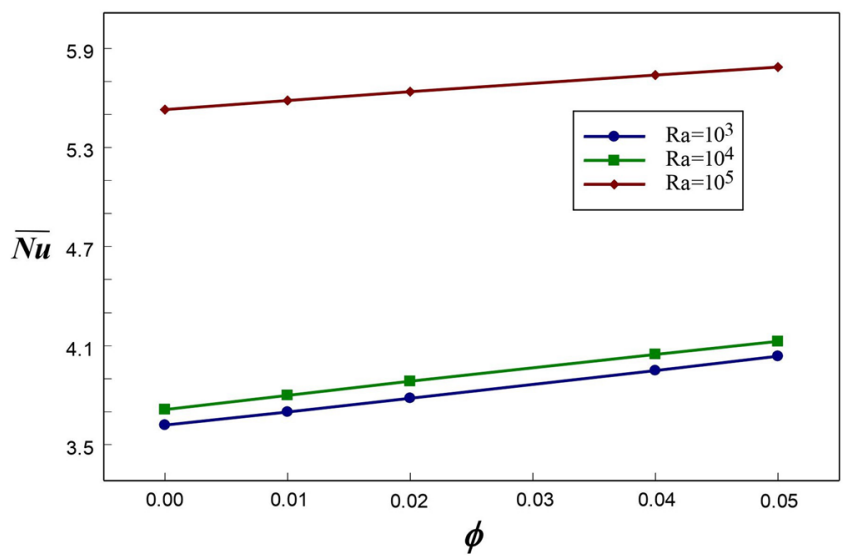

F I G URE 11 Influence of various $\phi$ and $R a$ on $\overline{N u}$ for $\gamma=\pi / 4, \varepsilon=0.75$ [Color figure can be viewed at wileyonlinelibrary.com] 

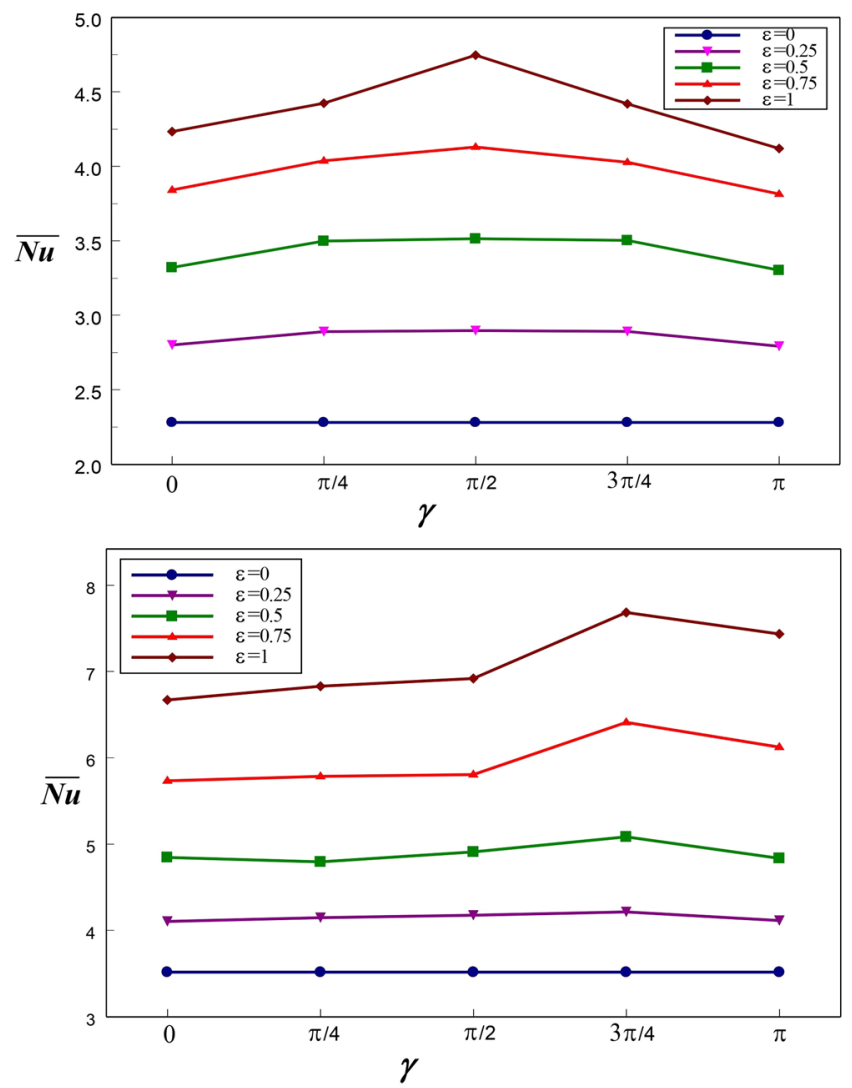

F I G URE 12 Effect of $\gamma$ and $\varepsilon$ on average Nusselt number for $R a=10^{3}$ (top), $R a=10^{5}$ (bottom) with $\phi=0.05$ [Color figure can be viewed at wileyonlinelibrary.com]

effectively enhanced with a careful selection of amplitude ratio and phase deviation for every $R a$.

In all heat transport processes caused by finite thermal gradients, the generation of irreversibility factor could not be avoided, which leads to degradation of system energy. This is quantified by means of the entropy generation whose magnitude reveals the efficiency of a thermal system. To understand the combined impacts of $R a$ and $\gamma$ on the total irreversibility and Bejan number, a parametric analysis has been performed and is portrayed in Figure 13. Figure 13 shows the effect of phase deviation and $R a$ on $S_{\text {tot }}$ and $B e$ for $\phi=0.05$ and $\varepsilon=0.25$. In general, total entropy generation enhances with the enhancement of the Rayleigh number. At a lower magnitude of $R a=10^{3}$, total entropy generation increase up to $\pi / 2$, and a further rise in phase deviation causes a marginal declination of the total entropy generation. For $R a>10^{3}, S_{t o t}$ is an increasing function of phase deviation. To apprehend the contribution of entropy due to heat over fluid friction entropy, the analysis of global Bejan number, $B e$, has been estimated by considering a broad range of $R a$ and $\gamma$. At lower Rayleigh number $\left(R a=10^{3}\right), B e>0.5$ at all phase deviations and this indicates the major contribution of irreversibility is from heat transfer rather than fluid drag. However, for $R a>10^{3}$, due to dominance of buoyancy force, fluid friction contributes major entropy as compared to thermal transport and can be witnessed through the magnitude of total Bejan number, $B e<0.5$ for 

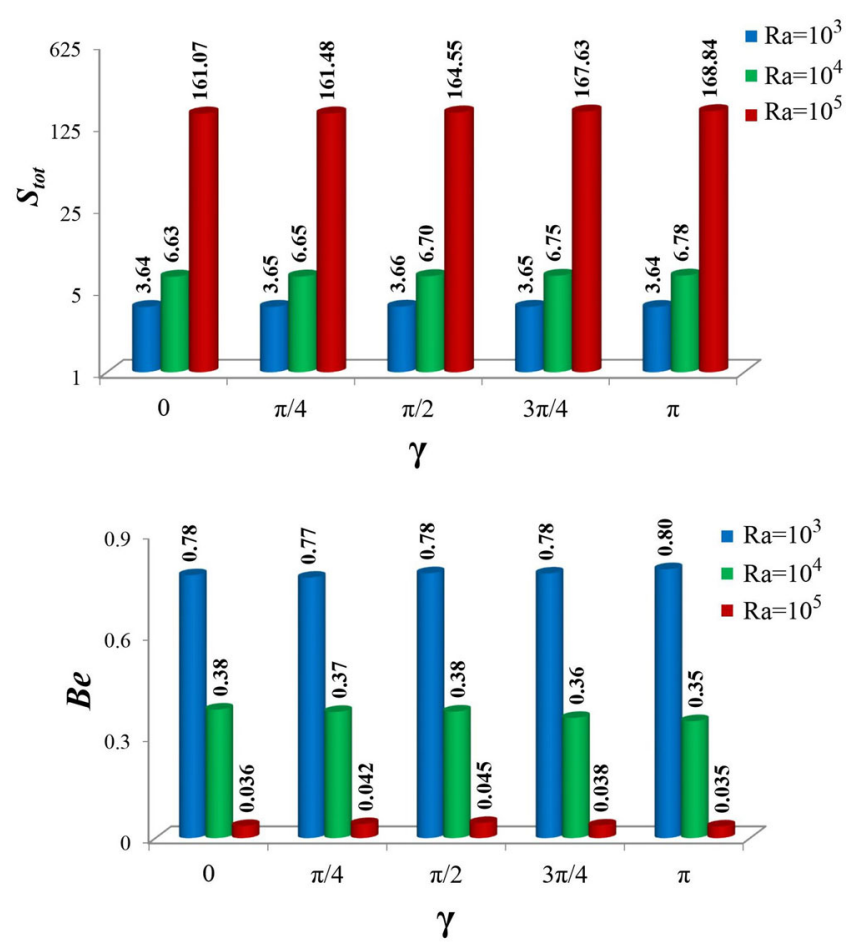

FIG URE 13 Effect of $\gamma$ and $R a$ on total entropy generation (top) and Bejan number (bottom) for $\varepsilon=0.25$, $\phi=0.05$ [Color figure can be viewed at wileyonlinelibrary.com]

$R a=10^{4}$ and $B e \ll 0.5$ for $R a=10^{5}$. In general, as the Bejan number is inversely proportional to $S_{\text {tot }}$, it decreases with an increase in $R a$.

Figure 14 reveals the variation of total entropy generation and Bejan number for different amplitude ratios and Rayleigh number with constant phase deviation and nanoparticle volume fraction. As discussed above, the impact of $R a$ on $S_{\text {tot }}$ and $B e$ is vividly witnessed through their respective magnitudes. In addition, irrespective of $R a$, an increase in amplitude ratio enhances total entropy generation and maximum entropy is produced for unit amplitude ratio. To identify the contribution of individual components to global entropy generation, the Bejan number is estimated for different magnitudes of $R a$ and $\varepsilon$. It is noticed that for the lower Rayleigh number $\left(R a=10^{3}\right)$ with $\varepsilon>0$, the Bejan number is higher and this demonstrates that $S_{T}$ is dominant. For the higher Rayleigh number $\left(R a=10^{5}\right), B e \ll 0.5$ for all amplitude ratios indicate that $S_{\Psi}$ is dominant.

The variation of $S_{\text {tot }}$ and $B e$ for different $\phi$ and $R a$ with fixed amplitude ratios and phase deviations are shown in Figure 15. As the nanofluid flow strength declines with increasing the nanoparticle concentration, fluid friction irreversibility reduces, which leads to a decrement in $S_{\Psi}$. For $R a=10^{3}$, the total entropy generation enhances with an increase in nanoparticle concentration, while for moderate $R a, S_{\text {tot }}$ declines with an increase in $\phi$ and it is also noticed that rather than nanofluid, base fluid generates less entropy with this choice of phase deviation and amplitude ratio for $R a=10^{5}$. On increasing nanoparticle volume fraction, Bejan number moves toward 1 and 0.5 , respectively, for $R a=10^{3}$ and $R a=10^{4}$, indicating that for lower Rayleigh number $S_{T}$ is dominant and for moderate $R a, S_{T}$ and $S_{\Psi}$ are of almost equal order of 

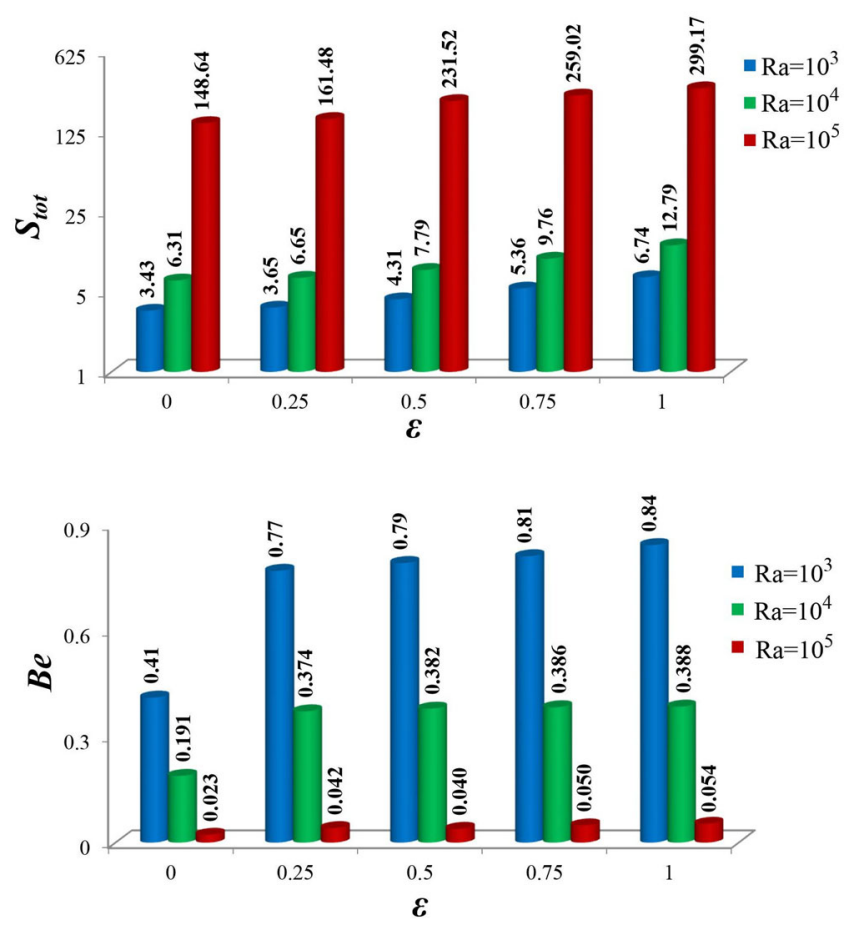

F I G U RE 14 Effect of $\varepsilon$ and $R a$ on total entropy generation (top) and Bejan number (bottom) for $\gamma=\pi / 4, \phi=0.05$ [Color figure can be viewed at wileyonlinelibrary.com]

magnitude. But for the larger Rayleigh number $\left(R a=10^{5}\right), B e \ll 0.5$, indicating the major influence of $S_{\Psi}$ on $S_{t o t}$ compared to $S_{T}$.

Figure 16 reveals the change in entropy produced due to heat transfer, fluid friction, and by both with varying phase deviation (top), amplitude ratio (middle), and volume fraction (bottom) by fixing other parameters. The phase deviation has a meager impact on entropy generation due to heat transfer but has a considerable effect on entropy generation from fluid friction, which leads to an enhancement of total entropy generation in the annular space. Entropy production due to heat transfer, fluid friction, and total are profoundly modified by $\varepsilon$ and $\phi$. As compared to phase deviation, amplitude ratio significantly alters the entropy production and thus it could be viewed as a vital parameter to control the entropy and thermal transport in the annular space. In our analysis, one of the objectives is to explore the influences of phase deviation and the ratio between the amplitudes on entropy production and is depicted in Figures 17 and 18 for low and high $R a$. The impacts of $\gamma$ and $\varepsilon$ for $R a=10^{3}$ and $\phi=0.05$ on global entropy production and Bejan number are shown in Figure 17. Total entropy generation is invariant at zero amplitude ratio for all $\gamma$ as a thermal condition at the outer cylinder is uniform. For all phase deviations, enhancement of amplitude ratio augments the entropy production and it can be noticed that the maximum entropy is generated for $\gamma=\pi / 2$. At all phase deviations with $\varepsilon>0$, the Bejan number is higher than 0.5, indicating that total entropy production is primarily from thermal transport as compared to fluid friction. But for the zero amplitude ratio, $B e$ is close to 0.5 , which reveals that total entropy generation has an equal contribution from both heat and friction. Figure 18 deals with the effect of phase deviation and amplitude ratio on total entropy generation and Bejan number at higher Rayleigh number 

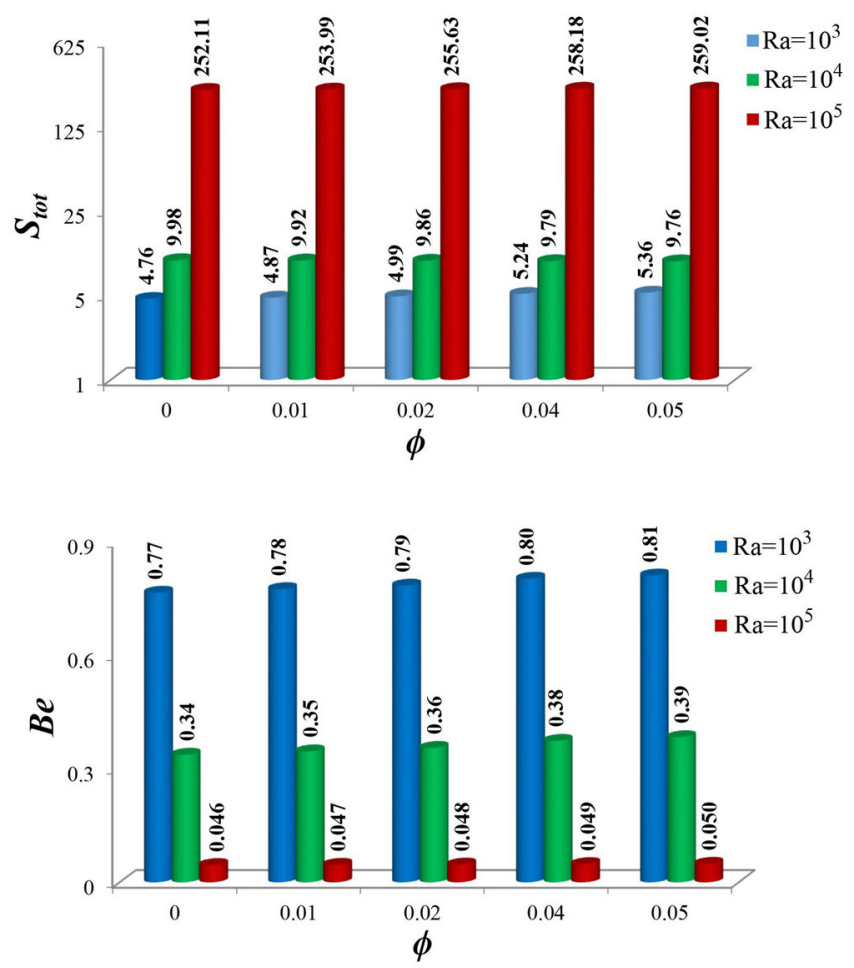

F I G U RE 15 Effect of $\phi$ and $R a$ on total entropy generation (top) and Bejan number (bottom) for $\gamma=\pi / 4, \varepsilon=0.75$. [Color figure can be viewed at wileyonlinelibrary.com]

$\left(10^{5}\right)$. For $\varepsilon=0, S_{t o t}$ and $B e$ are invariant with the enhancement of phase deviation. Minimum entropy generation is found at zero phase deviation for all amplitude ratios. Other than zero phase deviation, minimum entropy generation is gained at $\gamma=\pi / 4$ for $\varepsilon=0.25, \gamma=\pi / 2$ for $\varepsilon=0.5$, and at $\gamma=3 \pi / 4$ for $\varepsilon=0.75$, 1 . In all these cases, $B e \ll 0.5$ because higher $R a$ causes strong fluid flow, which leads to more $S_{\Psi}$.

The main purpose of this numerical experiment is to identify the parameter regimes at which maximum thermal energy should be dissipated with minimal production of entropy, which is desirable in any thermal system. This is achieved in our analysis through a parameter known as PEC, $\Gamma$, which is the ratio between global thermal dissipation rate and entropy production. In the current study, the major critical parameters are amplitude ratio, phase deviation, and nanoparticle concentration whose influences on $\Gamma$ are estimated and illustrated in Figure 19 for various reference samples of $\phi, \gamma$, and $\varepsilon$. In general, it is expected to have a higher value of PEC for a better thermal performance rate of the system. Figure 19 shows the PEC for various nanoparticle concentrations at three different phase deviations and amplitude ratios. The estimated PEC reveals that the enhancement of $\phi$ increases $\Gamma$, except at $\gamma=\pi / 4$ and $\varepsilon=0.5$. Among the various combinations of $\phi, \gamma$, and $\varepsilon$ considered, it has been found that the better thermal performance with less loss of system's energy is obtained for the choice of $\varepsilon=0.75, \gamma=3 \pi / 4$. Thus, it can be concluded that this choice of parameter is desirable in any thermal system to enhance the thermal dissipation rate at the cost of minimal entropy production. 

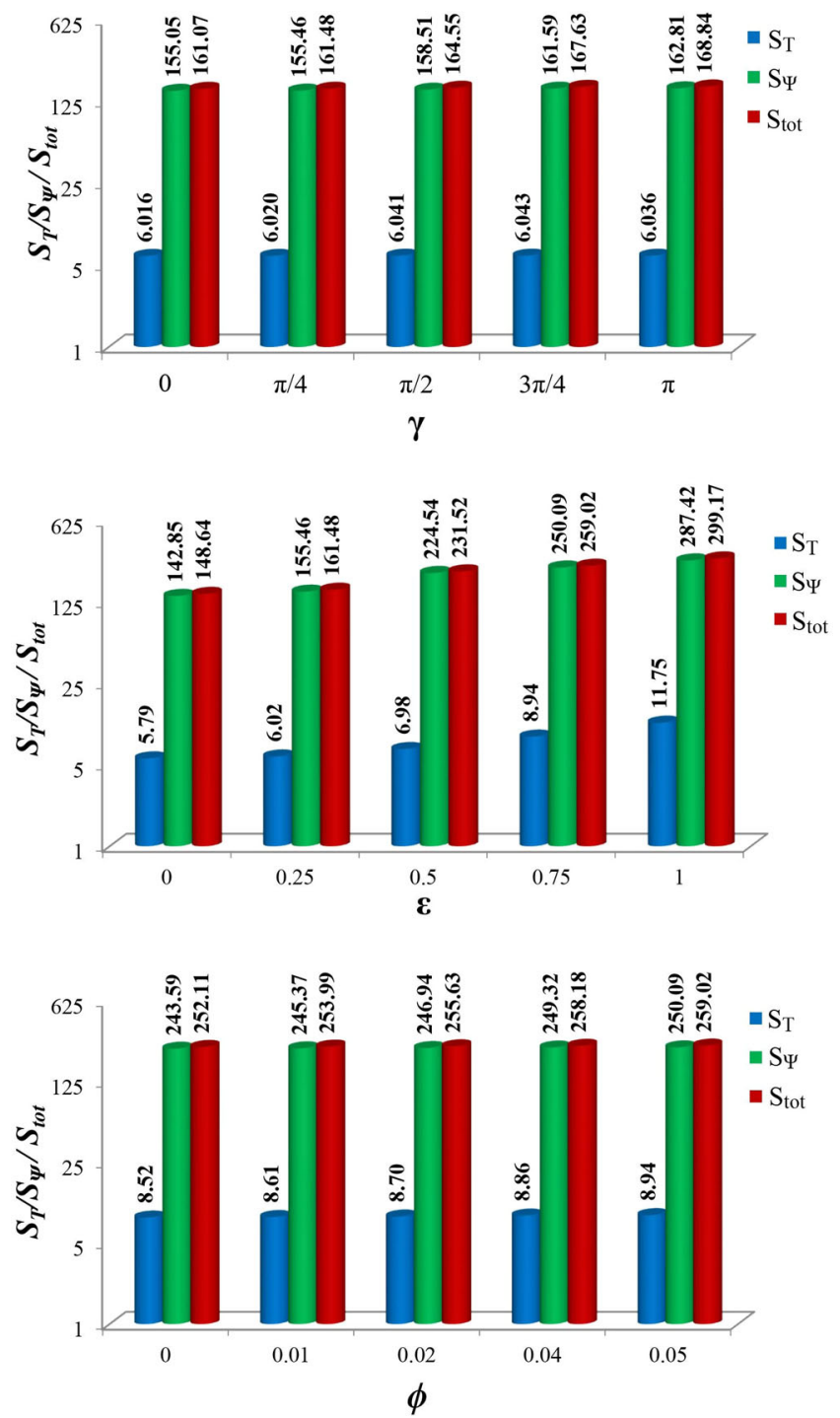

F I G U RE 16 Effect of $\gamma$ (top), $\varepsilon$ (middle), and $\phi$ (bottom) on $S_{T}, S_{\Psi}$, and $S_{\text {tot }}$ for $R a=10^{5}$ [Color figure can be viewed at wileyonlinelibrary.com]

\section{6 | CONCLUSIONS}

The current numerical study examines the buoyant thermal transport rate and entropy generation in an annular space filled with $\mathrm{Al}_{2} \mathrm{O}_{3}-\mathrm{H}_{2} \mathrm{O}$ nanofluid and is subjected to nonuniform sinusoidal thermal profiles having different phases deviation and amplitude. The impacts of major critical parameters on thermal dissipation and entropy generation are analyzed and important findings are summarized as follows:

1 The phase deviation induces a multicellular flow structure in the entire parameter regime. An increase in phase deviation reduces the number of vortices due to a change in the 

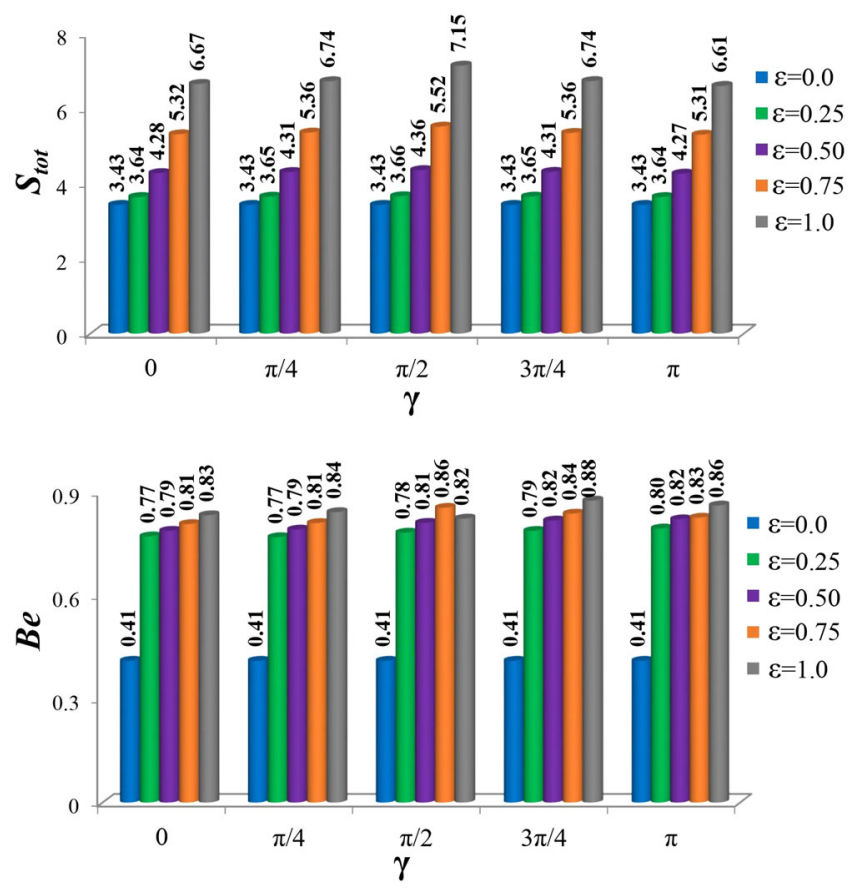

F I G U RE 17 Effect of $\gamma$ and $\varepsilon$ on total entropy generation (top) and Bejan number (bottom) for $R a=10^{3}, \phi=0.05$ [Color figure can be viewed at wileyonlinelibrary.com]
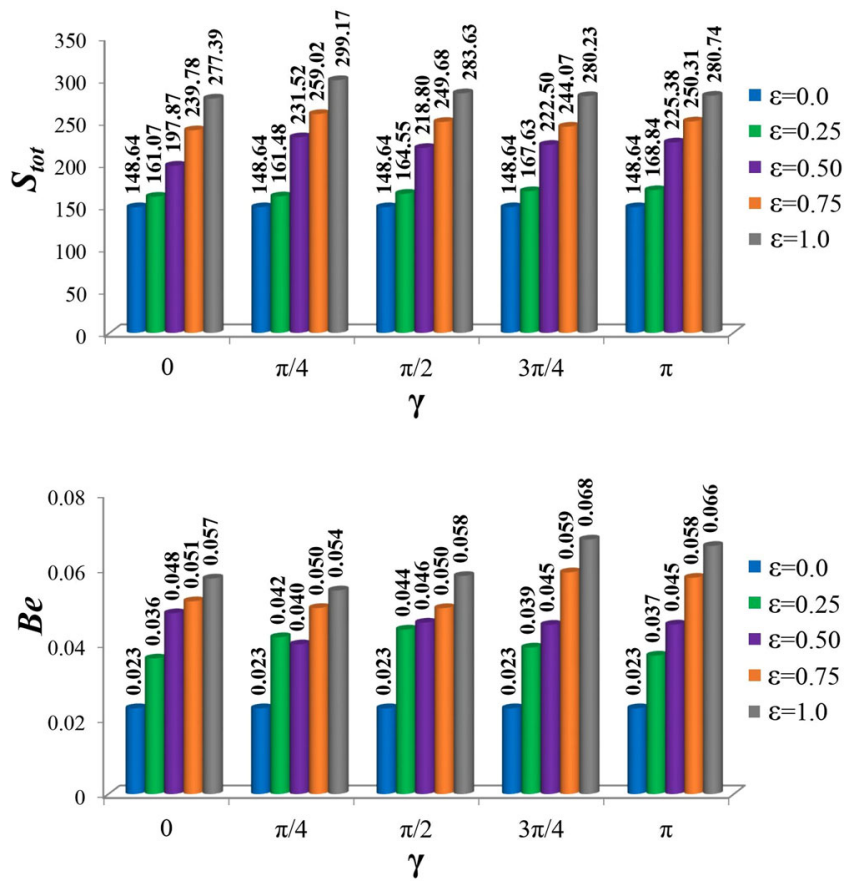

FIG URE 18 Effect of $\gamma$ and $\varepsilon$ on total entropy generation (top) and Bejan number (bottom) for $R a=10^{5}, \phi=0.05$ [Color figure can be viewed at wileyonlinelibrary.com] 

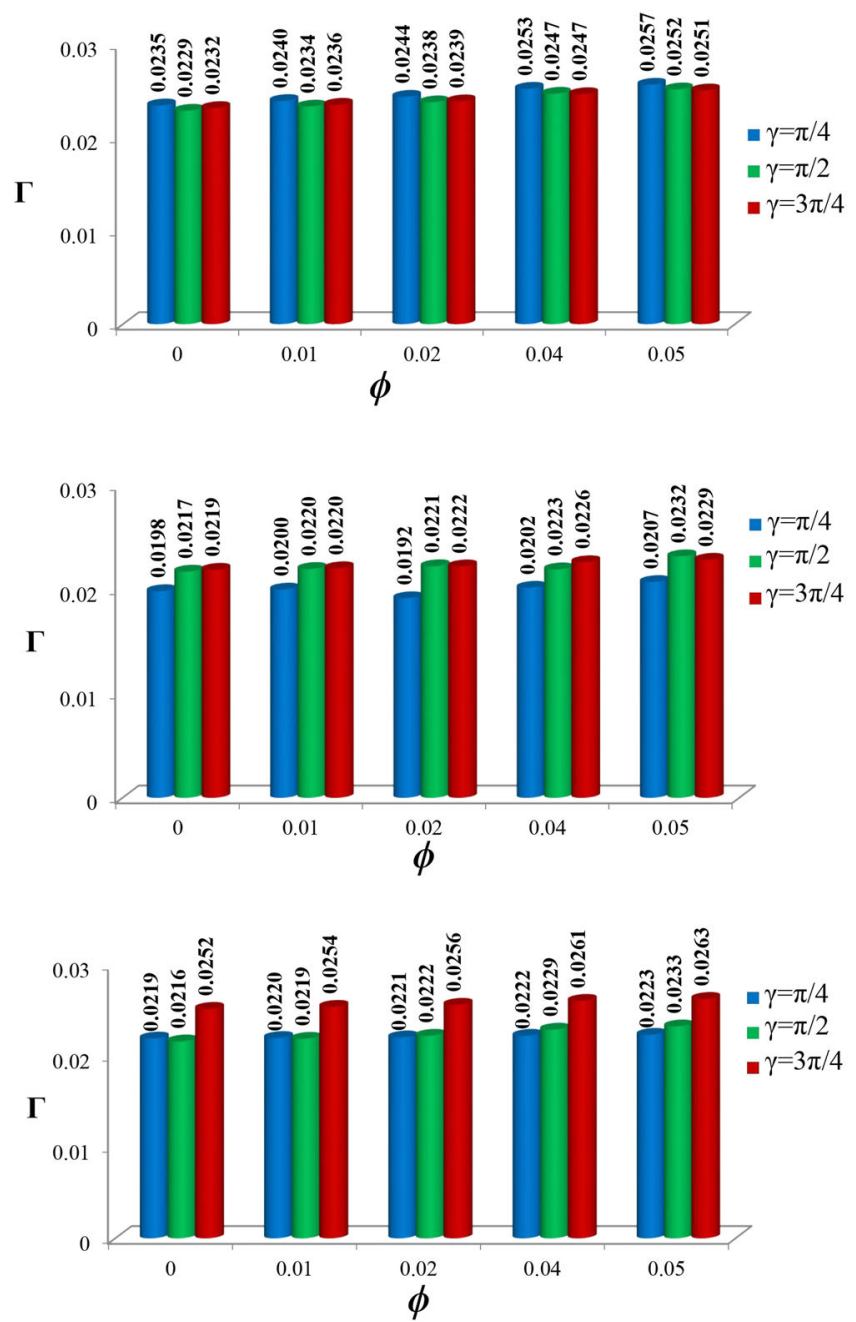

FIG URE 19 Effect of phase deviation and volume fraction on performance evaluation criteria $(\Gamma)$ with $R a=10^{5}$ for $\varepsilon=0.25$ (top), $\varepsilon=0.5$ (middle), and $\varepsilon=0.75$ (bottom) [Color figure can be viewed at wileyonlinelibrary.com]

temperature distribution on the outer cylinder. The fluid flow strength is high with symmetric bicellular flow compared to the four-cellular flow pattern.

2 For uniform cooling of the outer cylinder $(\varepsilon=0)$, a symmetrical flow with two eddies has been observed in the annular space. However, an increase in amplitude ratio alters the symmetric flow and increases the number of eddies by reducing the fluid flow strength. Furthermore, maximum heat produced from the inner cylinder is transferred to the cold region of the same wall.

3 The addition of nanoparticles to base fluid enhances the thermal transfer rate and reduces the fluid flow strength. Also, the thermal transport rate of nanofluid is an increasing function of Rayleigh number and amplitude ratio.

4 On enhancement of Rayleigh number from $10^{3}$ to $10^{5}$, the heat transfer rate is increased by $45.47 \%$ for phase deviation and $46.51 \%$ for amplitude ratio. 
5 At low $\left(R a=10^{3}\right)$ and moderate $\left(R a=10^{4}\right)$ Rayleigh numbers, where conduction is the dominant mode of heat transport, $\overline{\mathrm{Nu}}$ reaches a maximum for $\gamma=\pi / 2$ and at high Rayleigh number $\left(R a=10^{5}\right) \overline{N u}$ attains maximum for $\gamma=3 \pi / 4$.

6 It is observed that among the vast simulations performed, the maximum entropy is generated for $\varepsilon=1$ and $\gamma=\pi / 4$. Also, minimum entropy generation is recorded for lower magnitudes of phase deviation and amplitude ratio.

7 From the detailed simulations, for lower $R a=10^{3}$, entropy production is mainly due to $S_{T}$; however, for higher $R a=10^{5}$, fluid friction irreversibility plays a major role in entropy generation. In general, $S_{T}$ and $S_{\Psi}$ attain maximum and minimum values for a particular choice of $\gamma$ and $\varepsilon$, which also leads to the generation of maximum and minimum entropy, respectively.

8 As the Bejan number is inversely proportional to total entropy generation (Equation 12), increasing total entropy suppresses the Bejan number. For the lower Rayleigh number, $B e \gg 0.5$ indicates the dominance of $S_{T}$. In a similar way, for $R a=10^{5}, B e \ll 0.5$ indicates that $S_{\Psi}$ has maximum contribution to $S_{t o t}$.

9 The major finding of the present investigation is that the better thermal performance with minimum system energy loss is attained for the selection of $\gamma=3 \pi / 4$ and $\varepsilon=0.75$.

\section{ACKNOWLEDGMENTS}

M. Sankar and H. A. Kumara Swamy are supported by the Presidency University, Bengaluru and VGST. M. Sankar acknowledges the financial support provided by the VGST, GoK, under Grant Number KSTePS/VGST-KFIST (L1)/2017. Younghae Do was supported by the National Research Foundation of Korea (NRF) grant funded by the Korea Government (MSIP) (No. NRF-2019R1A2B5B01070579).

\section{CONFLICT OF INTEREST}

The authors declare that they have no conflict of interest.

\section{AUTHOR CONTRIBUTIONS}

M. Sankar and Younghae Do designed the research problem and developed the code. H. A. Kumara Swamy and Sebastian Altmeyer performed numerical simulations and plotted the graphs. H. A. Kumara Swamy and Sebastian Altmeyer analyzed the results and wrote the paper. M. Sankar and Younghae Do proofread the paper and managed the overall preparation.

\section{ORCID}

M. Sankar (1) http://orcid.org/0000-0001-5791-9964

H. A. Kumara Swamy (D) https://orcid.org/0000-0001-7422-7644

Younghae Do (D) http://orcid.org/0000-0003-3558-2349

Sebastian Altmeyer (D) http://orcid.org/0000-0001-5964-0203

\section{REFERENCES}

1. de Vahl Davis G, Thomas RW. Natural convection between concentric vertical cylinders. Phys Fluids. 1969; 12:198-207.

2. Prasad V, Kulacki FA. Free convection heat transfer in a liquid-filled annulus. ASME J Heat Transfer. 1985; 107:596-602.

3. Sankar M, Park Y, Lopez JM, Do Y. Double-diffusive convection from a discrete heat and solute source in a vertical porous annulus. Transp Porous Med. 2012;91:753-775. 
4. Wang S, Faghri A, Bergman TL. Transient natural convection in vertical annuli: numerical modeling and heat transfer correlation. Numer Heat Transfer A. 2012;61:823-836.

5. Sankar M, Do Y, Ryu S, Jang B. Cooling of heat sources by natural convection heat transfer in a vertical annulus. Numer Heat Transfer A. 2015;68:847-869.

6. Afrand M, Toghraie D, Karimipour A, Wongwises S. A numerical study of natural convection in a vertical annulus filled with gallium in the presence of magnetic field. J Magn Magn Mater. 2017;430:22-28.

7. Girish N, Sankar M, Makinde OD. Developing buoyant convection in vertical porous annuli with unheated entry and exit. Heat Transfer. 2020;49:2551-2576.

8. Pushpa BV, Sankar M, Makinde OD. Optimization of thermosolutal convection in vertical porous annulus with a circular baffle. Therm Sci Eng Prog. 2020;20:100735.

9. Choi SUS, Eastman JA. Enhancing thermal conductivity of fluids with nanoparticles. ASME International Mechanical Engineering Congress and Exhibition; 1995.

10. Putra N, Roetzel W, Das SK. Natural convection of nano-fluids. Heat Mass Transfer. 2003;39:775-784.

11. Abouali $\mathrm{O}$, Falahatpisheh A. Numerical investigation of natural convection of $\mathrm{Al}_{2} \mathrm{O}_{3}$ nanofluid in vertical annuli. Heat Mass Transfer. 2009;46:15-23.

12. Mabarek-Oudina F. Convective heat transfer of titania nanofluids of different base fluids in cylindrical annulus with discrete heat source. Heat Transfer. 2019;48:135-147.

13. Mabarek-Oudina F, Bessaih R. Numerical simulation of natural convection heat transfer of copper-water nanofluid in a vertical cylindrical annulus with heat source. Thermophys Aeromech. 2019;26:325-334.

14. Khan SA, Siddiqui MA. Numerical studies on heat and fluid flow of nanofluid in a partially heated vertical annulus. Heat Transfer. 2020;49:1458-1490.

15. Reddy NK, Sankar M. Buoyant convective transport of nanofluids in a non-uniformly heated annulus. J Phys Conf Ser. 2020;1597:012055.

16. Bilgen E, Yedder RB. Natural convection in enclosure with heating and cooling by sinusoidal temperature profiles on one side. Int J Heat Mass Transfer. 2007;50:139-150.

17. Deng Q, Chang J. Natural convection in a rectangular enclosure with sinusoidal temperature distributions on both sidewalls. Numer Heat Transfer A. 2008;54:507-524.

18. Bhuvaneswari M, Sivasankaran S, Kim YJ. Magnetoconvection in a square enclosure with sinusoidal temperature distribution on both side walls. Numer Heat Transfer A. 2011;59:167-184.

19. Ilis GG, Mobedi M, Sunden B. Effect of aspect ratio on entropy generation in a rectangular cavity with differentially heated vertical walls. Int Commun Heat Mass Transfer. 2008;35:696-703.

20. Chen S, Liu Z, Bao S, Zheng C. Natural convection and entropy generation in a vertically concentric annular space. Int $J$ Therm Sci. 2010; 49: 2439-2452.

21. Basak T, Kaluri RS, Balakrishnan AR. Effect of thermal boundary conditions on entropy generation during natural convection. Numer Heat Transfer A. 2011;59:372-402.

22. Zahmatkesh I. On the importance of thermal boundary conditions in heat transfer and entropy generation for natural convection inside a porous enclosure. Int J Therm Sci. 2008;47:339-346.

23. Varol Y, Oztop HF, Pop I. Entropy generation due to natural convection in non-uniformly heated porous isosceles triangular enclosures at different positions. Int J Heat Mass Transfer. 2009;52:1193-1205.

24. Basak T, Kaluri RS, Balakrishnan AR. Entropy generation during natural convection in a porous cavity: Effect of thermal boundary conditions. Numer Heat Transfer A. 2012;62:336-364.

25. Alsabery AI, Ismael MA, Chamkha AJ, Hashim I, Abulkhair H. Unsteady flow and entropy analysis of nanofluids inside cubic porous container holding inserted body and wavy bottom wall. Int J Mech Sci. 2021; 193:106161.

26. Armaghani T, Ismael MA, Chamkha AJ. Analysis of entropy generation and natural convection in an inclined partially porous layered cavity filled with a nanofluid. Can J Phys. 2017;95(3):238-252.

27. Mukhopadhyay A. Analysis of entropy generation due to natural convection in square enclosures with multiple discrete heat sources. Int Commun Heat Mass Transfer. 2010;37:867-872.

28. Kaluri RS, Basak T. Entropy generation due to natural convection in discretely heated porous square cavities. Energy. 2011;36:5065-5080.

29. Dashti MA, Safavinejad A. Optimal design with entropy generation minimization approach in combined natural convection with surface radiation in a two-dimensional enclosure. Heat Transfer. 2019;48: 4049-4073. 
30. Shahi M, Mahmoudi AH, Raouf AH. Entropy generation due to natural convection cooling of a nanofluid. Int Commun Heat Mass Transfer. 2011;38:972-983.

31. Alipanah M, Ranjbar AA, Farnad E, Alipanah F. Entropy generation of natural convection heat transfer in a square cavity using $\mathrm{Al}_{2} \mathrm{O}_{3}$-water nanofluid. Heat Transfer. 2014;44:641-656.

32. Sheikholeslami M, Ashorynejad HR, Rana P. Lattice Boltzmann simulation of nanofluid heat transfer enhancement and entropy generation. J Mol Liq. 2016;214:86-95.

33. Malik A, Bessaïh R, Oztop HF, Al-Salem K, Bayrak F. Natural convection and entropy generation in a nanofluid filled cavity with thick bottom wall: effects of non-isothermal heating. Int J Mech Sci. 2017;126: 95-105.

34. Mohammadpourfard M, Aminfar H, Zonouzi SA. Numerical investigation of the magnetic field effects on the entropy generation and heat transfer in a nanofluid filled cavity with natural convection. Heat Transfer. 2017;46:409-433.

35. Bezi S, Souayeh B, Ben-Cheikh N, Ben-Beya B. Numerical simulation of entropy generation due to unsteady natural convection in a semi-annular enclosure filled with nanofluid. Int J Heat Mass Transfer. 2018; 124:841-859.

36. Kashyap D, Dass AK. Two-phase lattice Boltzmann simulation of natural convection in a Cu-water nanofluid-filled porous cavity: effects of thermal boundary conditions on heat transfer and entropy generation. Adv Powder Technol. 2018;29:2707-2724.

37. Ferhi M, Djebali R, Ali-Kouz W, Abboudi S, Chamkha AJ. MHD conjugate heat transfer and entropygeneration analysis of MWCNT/waternanofluid in a partially heated divided medium. Heat Transfer. 2021;50:126-144.

38. Mejri I, Mahmoudi A, Abbassi MA, Omri A. Magnetic field effect on entropy generation in a nanofluidfilled enclosure with sinusoidal heating on both side walls. Powder Technol. 2014;266:340-353.

39. Malik S, Nayak AK. MHD convection and entropy generation of nanofluid in a porous enclosure with sinusoidal heating. Int J Heat Mass Transfer. 2017;111:329-345.

40. Abdel-Nour Z, Aissa A, Mebarek-Oudina F, et al. Magnetohydrodynamic natural convection of hybrid nanofluid in a porous enclosure: numerical analysis of the entropy generation. J Therm Anal Calorim. 2020; 141:1981-1992.

41. Aly AM. Mixing between solid and fluid particles during natural convection flow of a nanofluid-filled $\mathrm{H}$ shaped cavity with three center gates using ISPH method. Int J Heat Mass Transfer. 2020;157:119803.

42. Dagtekin I, Oztop HF, Bahloul A. Entropy generation for natural convection in $\Gamma$-shaped enclosures. Int Commun Heat Mass Transfer. 2007;34:502-510.

43. Parvin S, Chamkha AJ. An analysis on free convection flow, heat transfer and entropy generation in an odd-shaped cavity filled with nanofluid. Int Commun Heat Mass Transfer. 2014;54:8-17.

44. Dutta S, Goswami N, Biswas AK, Pati S. Numerical investigation of magnetohydrodynamic natural convection heat transfer and entropy generation in a rhombic enclosure filled with $\mathrm{Cu}$-water nanofluid. Int J Heat Mass Transfer. 2019;136:777-798.

45. Seyyedi SM, Dogonchi AS, Hashemi-Tilehnoee M, Waqas M, Ganji DD. Entropy generation and economic analyses in a nanofluid filled L-shaped enclosure subjected to an oriented magnetic field. Appl Therm Eng. 2020;168:114789.

46. Alsabery AI, Ismael MA, Chamkha AJ, Hashim I. Numerical investigation of mixed convection and entropy generation in a wavy-walled cavity filled with nanofluid and involving a rotating cylinder. Entropy. 2018;20:664.

47. Chamkha AJ, Jomardiani G, Ismael MA, Ghasemiasl R, Armaghani T. Thermal and entropy analysis in L-shaped non-Darcian porous cavity saturated with nanofluids using Buongiorno model: comparative study. Math Meth Appl Sci. 2020:1-16.

48. Oztop HF, Al-Salem K. A review on entropy generation in natural and mixed convection heat transfer for energy systems. Renew Sustain Energy Rev. 2012;16:911-920.

49. Mahian O, Kianifar A, Kleinstreuer C, et al. A review of entropy generation in nanofluid flow. Int J Heat Mass Transfer. 2013;65:514-532.

50. Huminic G, Huminic A. Entropy generation of nanofluid and hybrid nanofluid flow in thermal systems: a review. J Mol Liq. 2020;302:112533. 
51. Torabi M, Peterson GP, Torabi M, Karimi N. A thermodynamic analysis of forced convection through porous media using pore scale modeling. Int J Heat Mass Transfer. 2016;99:303-316.

52. Reddy NK, Swamy HAK, Sankar M. Buoyant convective flow of different hybrid nanoliquids in a nonuniformly heated annulus. Eur Phys J Spec Top. 2021;230:1213-1225.

How to cite this article: Sankar M, Swamy HAK, Do Y, Altmeyer S. Thermal effects of nonuniform heating in a nanofluid filled annulus: Buoyant transport versus entropy generation. Heat Transfer. 2021;1-30. https://doi.org/10.1002/htj.22342 